\title{
Transcriptomic analysis reveals the role of Oryza sativa FOUR LIPS in response to salt stress in rice
}

Chunxia Zhang ( $\nabla$ spring1984@163.com )

CAS Institute of Botany: Institute of Botany Chinese Academy of Sciences https://orcid.org/00000001-8525-8868

Jie Zhang

Institute of Botany Chinese Academy of Sciences

Huichao Liu

Institute of Botany Chinese Academy of Sciences

Xiaoxiao Qu

Institute of Botany Chinese Academy of Sciences

Junxue Wang

Institute of Botany Chinese Academy of Sciences

Qixiumei He

Institute of Botany Chinese Academy of Sciences

Junjie Zou

Institute of Botany Chinese Academy of Sciences

Kezhen Yang

Institute of Botany Chinese Journal of Plant Ecology: Institute of Botany Chinese Academy of Sciences

Jie Le

Institute of Botany Chinese Academy of Sciences https://orcid.org/0000-0001-8854-3031

\section{Research Article}

Keywords: RNA-seq, ChIP-seq, OsFLP, OsCDKB1;1, Rice, Salt stress

Posted Date: February 23rd, 2022

DOI: https://doi.org/10.21203/rs.3.rs-1354294/v1

License: (c) (1) This work is licensed under a Creative Commons Attribution 4.0 International License.

Read Full License 
1 Title: Transcriptomic analysis reveals the role of Oryza sativa FOUR LIPS in response to

2 salt stress in rice

\section{Authors:}

4 Chunxia Zhang ${ }^{1 *}$, Jie Zhang ${ }^{1,2 *}$, Huichao Liu $^{1,2}$, Xiaoxiao $\mathrm{Qu}^{1,2}$, Junxue Wang ${ }^{1,2,}$, Qixiumei

$5 \quad \mathrm{He}^{1,2}$, Junjie Zou ${ }^{1,3}$, Kezhen Yang ${ }^{1}$ and Jie Le ${ }^{1,2 \uparrow}$

6 Addresses:

$7 \quad{ }^{1}$ Key Laboratory of Plant Molecular Physiology, CAS Center for Excellence in Molecular Plant

8 Sciences, Institute of Botany, Chinese Academy of Sciences, Beijing 100093, China

$9 \quad{ }^{2}$ University of Chinese Academy of Sciences, Beijing 100049, China

$10{ }^{3}$ Biotechnology Research Institute, Chinese Academy of Agricultural Sciences, Beijing 100081,

11 China

$12{ }^{\dagger}$ Correspondence: Jie Le (lejie@ibcas.ac.cn), Tel: +86 1062836673

13 Orcid-ID: Jie Le, https://orcid.org/ 0000-0001-8854-3031

14 *These authors contributed equally to this work.

15 Present address:

16 §Wenbo School, Jinan 250100, China

17 Author e-mail addresses:

18 Chunxia Zhang: spring1984@163.com

19 Jie Zhang: jieer1027@163.com

20 Huichao Liu: liuhuichao@ibcas.ac.cn

21 Xiaoxiao Qu: quxiaoxiao721@sina.com

22 Junxue Wang: wangjunxues@163.com

23 Qixiumei He: heqixiumei@ibcas.ac.cn

24 Junjie Zou: zoujunjie@caas.cn

25 Kezhen Yang: ykzdsp@163.com

26 Jie Le: 1ejie@ibcas.ac.cn 
Key message: An R2R3-MYB transcription factor Oryza sativa FOUR LIPS associated with Oryza sativa B-type Cyclin-Dependent Kinase 1;1 confers salt tolerance in rice

Keywords: RNA-seq, ChIP-seq, OsFLP, OsCDKB1;1, Rice, Salt stress

\section{Abstract}

The Arabidopsis FOUR LIPS (AtFLP), an R2R3 MYB transcription factor, acts as an important stomatal development regulator. Only one orthologue protein of AtFLP, Oryza sativa FLP (OsFLP), was identified in rice. However, the function of OsFLP is largely unknown. In this study, we conducted RNA-seq and ChIP-seq to investigate the potential role of OsFLP in rice. Our results reveal that OsFLP is probably a multiple functional regulator involved in many biological processes in growth development and stress responses in rice. However, we mainly focus on the role of OsFLP in salt stress response. Consistently, phenotypic analysis under salt stress conditions showed that $O s f l p$ exhibited significant sensitivity to salt stress, while $O S F L P$ over-expression lines displayed obvious salt tolerance. Additionally, Yeast onehybrid assay and electrophoretic mobility shift assay (EMSA) showed that OsFLP directly bond to the promoter region of Oryza sativa B-type Cyclin-Dependent Kinase 1;1 (OsCDKB1;1), and the expression of $O s C D K B 1 ; 1$ was repressed in $O s f l p$. Disturbing the expression of $O s C D K B 1 ; 1$ remarkably enhanced the tolerance to salt stresses. Taken together, our findings reveal a crucial function of OsFLP regulating OsCDKB 1;1 in salt tolerance and largely extend the knowledge about the role of OsFLP in rice.

\section{Introduction}

MYB (myeloblastosis) transcription factor genes family have a wide function in plant adaptive responses to adverse environment and growth development (El-Kereamy et al. 2012; Lv et al. 2017; Yang et al. 2012; Ye et al., 2015). MYB genes family are classified into four groups, including 1R-MYB, 2R-MYB, 3R-MYB, and 4R-MYB. Among them, R2R3-MYB genes are more prevalent in plants compared to those in animals (Martin and Paz-Ares 1997; Dubos et al. 2010). In Arabidopsis, FOUR LIPS (AtFLP) is an atypical R2R3-MYB transcription factor as a regulator in stomatal development (Yang and Sack 1995; Lai et al. 2005). Besides this, AtFLP/AtMYB88 play important role in abiotic stress responses (Xie et al. 2010b), root gravitropism by regulating the transcription of PIN genes (Wang et al. 2015), as well as regulating female reproductive development (Makkena et al. 2012). In addition, AtFLP/AtMYB88 are widely expressed in various tissues (Lei et al. 2015). These reports indicate that AtFLP serves as a pleiotropic regulator participating in several aspects of biological processes in Arabidopsis. Recently, the 
homolog of AtFLP in rice OsFLP was knocked out via the genome editing system. In contrast to the role in Arabidopsis, OsFLP is required for the proper orientation of symmetric division of guard mother cells in rice (Wu et al. 2019). However, little is known about the function of OsFLP and the downstream targets. Salinity stress as a crucial environmental factor affects plant growth and development and leads to reduce crop yield (Kreps et al. 2002; Gupta and Huang 2014; Roy et al. 2014). To adapt to unfavorable environmental conditions, plants have evolved molecular mechanisms to protect themselves against stresses (Matsui et al. 2008). Thus, unraveling the salt stress-responsive mechanisms will be beneficial for improving plant salinity tolerance and subsequently increasing crop production to ensure food security for the rapid growth of the global population. Salinity stress causes several aspects of detrimental effects on plants. One of the most severe effects is the accumulation of $\mathrm{Na}^{+}$and $\mathrm{Cl}^{-}$, which leads to ion imbalance and inhibits the uptake of $\mathrm{K}^{+}$resulting in reduced productivity and even death (James et al. 2011). Hence, maintaining $\mathrm{Na}^{+} / \mathrm{K}^{+}$homeostasis in cells is vital for plants to survive under salt stress conditions. On the other hand, the production of ROS is enhanced and further leads to oxidative damages to the membranes, lipids, proteins (Mittler 2002). Many enzyme proteins such as superoxide dismutase (SOD), peroxidase (POD), catalase(CAT), glutathione peroxidase (GPX), ascorbate peroxidase (APX), are involved in ROS scavenging, and the activity of these proteins positively correlates with salt tolerance in plants (Mäkelä et al. 1999; Xia et al. 2014).

Besides AtFLP/AtMYB88, several genes have functions not only in controlling stomatal development but also in response to abiotic stress. For example, HIC, a negative regulator of stomatal development, controls stomatal density in response to elevated $\mathrm{CO}_{2}$ (Gray et al. 2000). The cold-responsive gene ICE 1 participates in stomatal differentiation (Kanaoka et al. 2008). The Arabidopsis receptor-like kinase ERECTA (ER) controls stomatal patterning (Shpak et al. 2005). Over-expression of ER remarkably increased heat tolerance in plants (Shen et al. 2015). Hence, these data implicate the cross-talk of the signal transduction pathways between stomatal development and abiotic stress.

Cyclin-dependent kinases (CDKs) mainly play roles in regulating cell division in plants (De Veylder et al. 2003; Dewitte and Murray 2003). In Arabidopsis, 12 CDKs have been identified with six categories (CDKA to CDKF) (Vandepoele et al. 2002) (28). B-type CDKs including B1-type (CDKB1;1 and CDKB1;2) and B2-type (CDKB2;1 and CDKB2;2) subgroups are unique in plants (Vandepoele et al. 2002; Boudolf et al. 2001). In Arabidopsis, CDKB1;1 and CDKB1;2 act redundantly in regulating the 
symmetric division of Guard Mother Cells (GMCs). In addition, FLP/MYB88 could directly bind to the promoter region of $C D K B 1 ; 1$ to regulate its expression during stomatal development (Xie et al. 2010a). Moreover, CDKB1;1 together with the RPA2a, which is a subunit of replication protein A complexes, restricts stomatal proliferation by CDKB1;1-mediated phosphorylation (Yang et al. 2019). OsCDKB1;1 could restore the impaired GMC division and stomatal production in Atcdkb1;1 1;2 mutants (Qu et al. 2018). In addition, salt stress affects the cell cycle regulation at the transcriptional level, ultimately leading to adaptive growth responses (Burssens et al. 2000). Salt stress responses involve the decline of cell cycle activity in the meristem of the root (West et al. 2004). However, the involvement of OsCDKB 1;1 in response to abiotic stress, especially salt stress, is still unknown yet.

In this study, we isolated an Osflp mutant and generated the complemental transgenic lines. Using these materials, we performed the integrative analysis of RNA-seq and ChIP-seq. We found that the potential role of OsFLP was involved in several aspects of biological processes, especially in salt stress responses. Osflp was more sensitive to salt stresses with more ROS accumulation in plants compared with wild-type ZH11. Additionally, OsFLP could directly target the promoter region of OsCDKB1;1 in vitro and repress its transcriptional expression. Consistently, OsCDKB1;1-RNAi plants exhibited significant salt tolerance with less ROS accumulation. Collectively, we conclude that upon salt stresses, OsFLP represses the expression of $O s C D K B 1 ; 1$ to confer salt tolerance in rice. These findings provide new functions of OsCDKB1;1 out of cell cycle and novel insights into the role of OsFLP in rice.

\section{Materials and Methods}

\section{Plant Materials and growth conditions}

Rice plants, Oryza sativa L. ssp. Japonica cv. Zhonghua 11 (ZH11, wild type), were cultivated in the field of the Institute of Botany, Chinese Academy of Sciences, Beijing for propagating seeds. Plant growth chambers were also used for rice cultivating with the following setting: $12 \mathrm{~h}$ day at $30^{\circ} \mathrm{C}$ $\pm 2{ }^{\circ} \mathrm{C}, 12 \mathrm{~h}$ night at $22^{\circ} \mathrm{C} \pm 2{ }^{\circ} \mathrm{C}$, and $60 \%$ humidity.

Osflp mutant was isolated by TILLING (Targeting Induced Local Lesions IN Genomes) through an in-house TILLING platform (www.croptilling.org), which had a $\mathrm{G} \rightarrow \mathrm{A}$ point mutant in the sixth exon at the 2126 bp position downstream of ATG position which led to the substitution of Ala with Thr.

To generate $O s F L P$ and $O S C D K B 1 ; 1$ overexpression lines, the full-length cDNA fragments were amplified by PCR and were inserted into TOPO vector, afterward were sub-cloned into pH7WG2D.1 
vector via LR reaction of gateway cloning with $35 \mathrm{~S}$ promoter. To obtain the $O s f l p$ complementation line, 4733bp genomic fragment of OSFLP was amplified and was sub-cloned into the destination vector pCAMBIA1301. For making the GUS line, the promoter region of $O S F L P$ was fused to the vector pGUS1301. These constructs were introduced into rice via an Agrobacterium-mediated transformation (Hiei et al. 1994). The homozygous lines were selected for further analysis.

\section{Germination rates assay under salt stress}

127 Salt tolerance phenotype assay on half-strength Murashige \& Skoog medium (MS) solid medium plates.

128 Three-day-old rice seedlings grown on $1 / 2$ MS solid medium vertical plates were transferred to new $1 / 2$ MS solid medium vertical plates supplemented with or without $200 \mathrm{mM} \mathrm{NaCl}, 5$ days later taking pictures and performing measurements.

Salt tolerance phenotype assay using hydroponic culture. 14-day-old rice seedlings cultivated with $1 / 2$ MS hydroponic culture were transferred to liquid medium supplemented with or without $150 \mathrm{mM} \mathrm{NaCl}$ for 3 days or 10 days, changing liquid medium every 3 days, afterward transferred to fresh liquid medium for 3 days recovery.

Salt tolerance phenotype assay with rice seedlings grown on soil. 14-day-old rice seedlings grown on 1/2 MS solid medium were transferred to soil growing for 2 weeks and stopped watering for one week, afterward watering plants with tap water supplemented with or without $200 \mathrm{mM} \mathrm{NaCl}$ for 15 days and 21 days.

\section{Survival rates and soluble sugar content measurement.}

Survival rates were calculated after 21 days of salt stress treatment. No green shoots were counted as dead plants and the survival rate was shown as the percentage of alive seedlings.

Soluble sugar content was determined referring to the previous report (Tang et al. 2013). $500 \mathrm{mg}$ leaves were ground in $2 \mathrm{~mL} \mathrm{10 \%} \mathrm{trichloroacetic} \mathrm{acid} \mathrm{(TCA),} \mathrm{and} \mathrm{the} \mathrm{mixture} \mathrm{was} \mathrm{centrifuged} \mathrm{at} 4000$

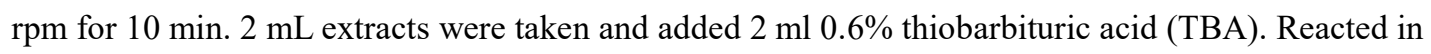
boiled water for $15 \mathrm{~min}$ and quickly cooled on ice, afterward centrifuged at $4000 \mathrm{rpm}$ for $15 \mathrm{~min}$. The spectral absorbance of the supernatant was determined at $450 \mathrm{~nm}, 532 \mathrm{~nm}$, and $600 \mathrm{~nm}$ with an ultraviolet spectrophotometer. Soluble concentration $(\mathrm{mM})=11.71 \times \mathrm{D} 450$. 
potassium phosphate buffer $(\mathrm{pH}$ 7.8) containing $1 \%$ polyvinylpyrrolidone was added and mixed thoroughly. Then the mixture was centrifuged at $15000 \mathrm{~g}$ at $4{ }^{\circ} \mathrm{C}$ for $20 \mathrm{~min}$. The supernatant was the crude enzyme extraction. The activities of SOD, POD, and CAT were determined following the previously described protocols (Miao et al. 2010). The final enzyme activities were calculated according to the following formulations. SOD activity $(\mathrm{U} / \mathrm{g} \cdot \min )=\left(\mathrm{A}_{0}-\mathrm{As}_{\mathrm{S}}\right) \times \mathrm{V}_{\mathrm{t}} \div\left(\mathrm{A}_{0} \times \mathrm{W} \times 0.5 \times \mathrm{V}_{\mathrm{s}}\right),\left(\mathrm{A}_{0}\right.$, absorbance value of the control; $\mathrm{A}_{\mathrm{S}}$, absorbance value of the samples; $\mathrm{V}_{\mathrm{t}}$, the total volume of the sample; $\mathrm{W}$, the fresh weight of the sample; $\mathrm{V}_{\mathrm{s}}$, the volume of the sample for measurement). POD activity $(\mathrm{U} / \mathrm{g} \cdot \mathrm{min})$ $=\left(\Delta \mathrm{A}_{470} \times \mathrm{V}_{\mathrm{t}}\right) \div(\mathrm{W} \times \mathrm{Vs} \times 0.01 \times \mathrm{t}), \quad\left(\Delta \mathrm{A}_{470}\right.$, the change of absorbance value at $470 \mathrm{~nm}$ wavelength per minute; $\mathrm{V}_{\mathrm{t}}$, the total volume of the sample; $\mathrm{W}$, the fresh weight of the sample; $\mathrm{V}_{\mathrm{s}}$, the volume of the sample for measurement; $\mathrm{t}$, interval time $)$. CAT activity $(\mathrm{U} / \mathrm{g} \cdot \min )=\left(\Delta \mathrm{A}_{240} \times \mathrm{V}_{\mathrm{t}}\right) \div(\mathrm{W} \times \mathrm{Vs} \times 0.1 \times \mathrm{t})$, $\Delta \mathrm{A}_{240}=\mathrm{A}_{\mathrm{S0}}-\left(\mathrm{A}_{\mathrm{S} 1}+\mathrm{A}_{\mathrm{S} 2}\right) / 2,\left(\Delta \mathrm{A}_{240}\right.$, the change of absorbance value at $240 \mathrm{~nm}$ wavelength per minute; $\mathrm{V}_{\mathrm{t}}$, the total volume of the sample; $\mathrm{W}$, the fresh weight of the sample; $\mathrm{V}_{\mathrm{s}}$, the volume of the sample for measurement; $t$, interval time; $\mathrm{A}_{\mathrm{S} 0}$, absorbance value of the control; $\mathrm{A}_{\mathrm{S} 1}$ and $\mathrm{A}_{\mathrm{S} 2}$, absorbance value of the samples).

\section{$\mathrm{Na}^{+}$and $\mathrm{K}^{+}$content assay}

Shoots and roots of rice seedlings were harvested separately. Samples were dried at $104{ }^{\circ} \mathrm{C}$ for $15 \mathrm{~min}$ and $55^{\circ} \mathrm{C}$ for 3 days. Dry samples were digested in nitric acid overnight in the dark and then transferred to $120^{\circ} \mathrm{C}$ for $8-12 \mathrm{~h}$. The content of $\mathrm{Na}^{+}$and $\mathrm{K}^{+}$in the extractions was measured with an ICP emission spectrometer (iCAP6300, Thermo scientific, US) according to the methods described previously (Shen et al. 2015b).

\section{ROS content determination}

171 The content of $\mathrm{H}_{2} \mathrm{O}_{2}$ was determined referring to the protocol described previously (53). The commercial

$172 \mathrm{H}_{2} \mathrm{O}_{2}$ detection kit (SolarBio Inc.) was used. In brief, approximately $100 \mathrm{mg}$ rice leaves were ground 173 with liquid nitrogen and $1 \mathrm{ml}$ lysate buffer was added and mixed gently. The extraction was centrifuged at $8000 \mathrm{~g}$ for $10 \mathrm{~min}$ at $4{ }^{\circ} \mathrm{C}$. The reaction buffer was added to the supernatant. Then mixed them gently,

175 and reacted for $5 \mathrm{~min}$ at room temperature. The mixture was measured with an ultraviolet spectrophotometer at the $415 \mathrm{~nm}$ wavelength, and the $\mathrm{H}_{2} \mathrm{O}_{2}$ content was calculated according to the

177 formulation in the manufacturer's protocol. We also used 3,3-diaminobenzidine (DAB) staining for the 178 detection of $\mathrm{H}_{2} \mathrm{O}_{2}$ and nitroblue tetrazolium (NBT) staining to determine $\mathrm{O}_{2}{ }^{-}$according to the previously 

into around $1 \mathrm{~cm}$ length, immersed in $1 \mathrm{mg} / \mathrm{mL}$ DAB solution, incubated for $8 \mathrm{~h}$ at room temperature. Afterward, samples were transferred into ethanol to remove the chlorophyll heating with a water bath at $100{ }^{\circ} \mathrm{C}$ for $2 \mathrm{~h}$. For NBT staining assay, around $1 \mathrm{~cm}$ length of rice leaves was immersed in $6 \mathrm{mM}$ NBT solution and incubated for 8 hours at room temperature. Ethanol was used to remove the chlorophyll and pictures were taken for analysis.

\section{Yeast one-hybrid assay}

In the yeast one-hybrid assay, the activated domain $\mathrm{AD}$ vector and the LacZ reporter $\mathrm{BD}$ vector were used. OsFLP was fused to the $\mathrm{AD}$ vector (OSFLP-AD). The 2266 bp promoter of $O s C D K B 1 ; 1$ was truncated into nine fragments (-2266 bp to $-1132 \mathrm{bp},-2266 \mathrm{bp}$ to $-1683 \mathrm{bp},-1701 \mathrm{bp}$ to $-1132 \mathrm{bp},-1208$ bp to -537 bp, -632 bp to -1 bp, -536 bp to -413 bp, -418 bp to -275 bp, -274 bp to -138 bp, -137 bp to $1 \mathrm{bp}$ ), and fused with LacZ reporter BD vector, respectively. Afterward, the fused $\mathrm{AD}$ and $\mathrm{BD}$ vectors were co-transformed into the EGY48 yeast strain and performed X-gal staining.

RNA-sequencing, ChIP-sequencing, and RT-qPCR analysis

For RNA-sequencing analysis, ZH11 and $O s f l p$ mutant plants were grown under $30^{\circ} \mathrm{C} \pm 2{ }^{\circ} \mathrm{C}, 12 \mathrm{~h}$ day / $22{ }^{\circ} \mathrm{C} \pm 2{ }^{\circ} \mathrm{C}, 12 \mathrm{~h}$ night conditions for 7 days. The shoots of the seedlings were harvested and frozen in liquid nitrogen. RNA was extracted with TRIzol reagent (Invitrogen). The libraries were sequenced based on the Illumina platform and were performed by BioMarker Inc. (Beijing, China). The clean reads were used for the subsequent analysis. Genes with adjusted p-value $<0.05$ and $\log 2$ fold- change $>1$ were taken as the differential expressed genes (DEGs). The GO enrichment of DEGs analysis was conducted with AgriGO V2.0 (http://systemsbiology.cau.edu.cn/agriGOv2/).

To generate $O s F L P: g O s F L P$-MYC plants, the genomic sequence of $O s F L P$ was amplified with the gOsFLP-F primer (ATGGCGACCGGACCGATCT) and gOsFLP-R primer (AAGCTTTTATACCGAGTTCAAGTCT), and sub-cloned into a pSUPER:1300-MYC vector driven with OsFLP native promoter. Chromatin immunoprecipitation(ChIP) was performed with 7-day-old rice seedlings. The sequencing was performed by Wuhan Igenebook Biotechnology Co., Ltd (Wuhan, China). Ltd). The first-strand cDNA was synthesized with a PrimeScript ${ }^{\circledR}$ RT Reagent Kit (Takara). Real-time 
volume contained the following ingredients: $10 \mu 1$ Hieff ${ }^{\text {qPCR }}$ SYBR ${ }^{\circ}$ Green Master Mix (No Rox ), $0.8 \mu 1$ gene-specific primers $(10 \mu \mathrm{M}), 2 \mu \mathrm{l}$ cDNA which had been diluted 10 times from the original cDNA solution. Three steps amplification program was used as follows: $95{ }^{\circ} \mathrm{C}$ for $5 \mathrm{~min}, 40$ cycles with $95{ }^{\circ} \mathrm{C}$ for $10 \mathrm{sec}, 55^{\circ} \mathrm{C}$ for $20 \mathrm{sec}$, and $72{ }^{\circ} \mathrm{C}$ for $20 \mathrm{sec}$, finally following with melting curve step. OsACT1 was used as the internal reference gene for normalization. The primers are listed in Table S3.

\section{Electrophoretic mobility shift assays (EMSA)}

The coding sequence of $O S F L P$ was amplified by PCR inserted into the pET28a vector and transformed into E. coli DE3 (BL21) cells for expressing the recombinant protein. The OsFLP protein was purified with His tag. Oligonucleotide probes were synthesized and labeled with Biotin 3' End DNA Labeling Kit (PIERCE). For EMSA assay, $20 \mu \mathrm{L}$ volume of reactions included the following components: His-OsFLP recombinant protein, labeled probes, 10×binding buffer, $100 \mathrm{mM} \mathrm{MgCl}_{2}, 50 \%$ (v/v) glycerol, $1 \mu \mathrm{g} / \mu \mathrm{L}$ poly $(\mathrm{dI}-\mathrm{dC})$, and $1 \%(\mathrm{v} / \mathrm{v})$ Nonidet $\mathrm{P}-40$. Unlabeled probes were added for the competition assay. The reaction was incubated at room temperature for $20 \mathrm{~min}$. The reaction mixtures were analyzed by electrophoresis. Te LightShift Chemiluminescent EMSA kit (Thermo Scientific, 20148) was used to detect the Biotin-labeled probes.

\section{Results}

\section{The phylogenetic relationship analysis of FLP in plants}

The FLP encodes an R2R3-MYB transcription factor protein (Lai et al. 2005; Wu et al. 2019). To explore the evolutionary relationship of the FLP in different species, including algae, lower plants, and higher plants, we conducted phylogenetic tree analysis with OsFLP amino acids as a probe. The results revealed that the OsFLP orthologous proteins were not only present in higher plants (Panicum miliaceum, Brachypodium distachyon, Setaria viridis, Setaria italic, Zea mays, Sorghum bicolor, Oryza sativa, Arabidopsis thaliana, Glycine max, Medicago truncatula, and Medicago sativa) with stomata but also in the lower plants (Zostera marina) without stomata, even in the single-cell green algae (Coccomyxa subellipsoidea and Chlamydomonas reinhardtii) (Fig.S1). Thus, these data indicate that besides the

233 function in regulating stomatal development, FLP may be also involved in other functions in plant development or adaptation to unfavorable environmental conditions.

\section{Transcriptomic analysis of the $O$ sflp mutant}

To facilitate functional analysis of OsFLP, we isolated an Osflp mutant line based on TILLING system. 

in the mature leaves. The genomic region of $O S F L P$ driven with the native promoter was introduced into the $O s f l p$. Several complementation lines were obtained and the abnormal GCs phenotype was rescued in these lines. One complementation line was used in this study and indicated as COM (Supplementary

241 Fig. 1A-E). This phenotype is consistent with the previous report on Osflp created by CRISPR (Wu et al. 242 2019).

243 To explore the potential functions of OsFLP, we performed the RNA-sequencing (RNA-seq) analysis 244 with the shoots of seven-day-old seedlings of Osflp and the wild-type ZH11 under normal growth conditions. In total, we identified 755 differentially expressed genes (DEGs), including 328 up-regulated genes and 427 down-regulated genes in Osflp compared with ZH11 (Fig. 2A, Dataset S1). In these DEGs, several types of genes might be regulated by OsFLP including "transcription factors" "transferase proteins" "cell wall-related genes" "environmental stress-responsive genes" "metabolism pathway" and "hormone-related genes", etc. (Fig. 2B, Dataset S2). These results indicate that OsFLP may be involved in various aspects of plant development and environmental stress responses.

To further unravel the function of OsFLP, we performed Gene Ontology (GO) analysis with upregulated and down-regulated DEGs, respectively. Intriguingly, GO analysis revealed that GO terms related to peroxidase activity, oxidative stress reactive oxygen species metabolic process, and so on were significantly enriched with down-regulated DEGs other than up-regulated DEGs (Fig. 2C, Dataset S3). Several class III peroxidases (PRXs) were identified according to the GO terms and most of these PRXs were downregulated in Osflp (Supplementary Table S1). Furthermore, to confirm the RNA-seq data, the expression of four randomly selected genes was detected by RT-qPCR. The results are consistent with RNA-seq (Supplementary Fig. S2A). Considering PRXs responsible for $\mathrm{H}_{2} \mathrm{O}_{2}$ metabolism (Almagro et al. 2009), OsFLP may play a role in controlling ROS-related pathways. Thus, the expression of POD1, POD2, and CAT, which is involved in ROS scavenging (Miller et al. 2010; Mittler 2002) (Noctor and Foyer 1998), were detected. The results showed that the expression of three genes significantly decreased in Osflp compared with ZH11 (Supplementary Fig. S2B).

To further identify the direct targets of OsFLP, the ChIP-seq analysis was conducted with pOsFLP::OsFLP-MYC in Osflp. After removal of the background peaks, a total of 12899 peaks were 
to various pathways, including "spliceosome", "mRNA surveillance pathway", "N-Glycan biosynthesis", "endocytosis", "RNA transport” and "oxidative phosphorylation", etc. (Supplementary Fig. S3, Dataset S5). Among them, oxidative phosphorylation is closely related to ROS metabolism.

Through the integrative analysis of RNA-seq data and ChIP-seq data, 125 genes were found to be present in both RNA-seq and ChIP-seq profiles (Fig. 2D, Dataset S6). KEGG pathway analysis showed that peroxisome related pathway was included (Supplementary Fig. S4, Dataset S7). Peroxisome mainly controlling ROS scavenging was enriched. This result implied that OsFLP may play a role in ROS metabolism.

In addition, 10 genes out of 125 genes were related to salt stress responses, $\mathrm{Na}^{+}$and $\mathrm{K}^{+}$transports, as well as oxidoreductase activity (Supplementary Table S2). In addition, to further confirm the salt stressresponsive function of $O S F L P$, the expression levels of four salt stress-responsive genes including OsRab16A, OsNAC5, OsDREB2A, and OsDREB1F (Song et al. 2011; Ponce et al. 2021; Yang et al. 2012), were examined in ZH11, Osflp, COM, and OE with or without $\mathrm{NaCl}$ stress. With salt stress conditions, the expression of these four genes significantly decreased in Osflp, whereas increased in OE, compared with ZH11 and COM in rice (Fig. 2E-H). Taken together, these data show that OsFLP affects the expression of salt stress-responsive genes as well as the ROS metabolic genes, suggesting the possible involvement of OsFLP in salt stress responses.

\section{$O S F L P$ plays a vital role in response to salt stress}

To verify the role of OsFLP in response to salt stress, the expression pattern of $O S F L P$ under salt stresses was determined. The expression of $O S F L P$ was increased after $2 \mathrm{~h}$ salt stress treatment and reached the maximal level after $6 \mathrm{~h}$ salt stress treatment (Supplementary Fig. S5A). Consistently, the shoots and leaves of the $p O s F L P::$ GUS transgenic plants also showed increased GUS activity after $6 \mathrm{~h}$ salt stress treatment (Supplementary Fig. S5B).

The increased expression of $O S F L P$ induced by salt stress encouraged us to examine the phenotype of $O s f l p$ under salt stresses. Upon $150 \mathrm{mM} \mathrm{NaCl}$ stress, the germination rate of ZH11 and COM were 291 almost 100\%, while the germination rate of Osflp was much lower, around 53\% after 5 days (Supplementary Fig. S6A, B, and C). Furthermore, under $200 \mathrm{mM} \mathrm{NaCl}$ stress conditions, compared to 293 ZH11 and COM, Osflp had shorter roots and shoots. In contrast, significant increases in shoot and root length were observed in the OSFLP over-expression plants (OE) (Fig. 3A - C). To further confirm the 
salt responsive function of $O S F L P$, we performed the phenotypic analysis with plants cultivated in soil.

Three-week-old seedlings were treated with $200 \mathrm{mM} \mathrm{NaCl}$ for 15 days and 21 days, respectively. Osflp exhibited more sensitivity with the significantly decreased survival rate (about 18.75\%) compared with ZH11(about 87.5\%), and COM plants could completely rescue the salt-sensitive phenotype with around $81.25 \%$ of plants survival rate, whereas OE plants behaved more resistance to salt stress with $100 \%$ survival rate (Fig. 3D and E). Taken together, these data indicate that OsFLP serves as a positive regulator in salt stress response in rice.

\section{Osflp accumulates more ROS under salt stress conditions}

Salt stress usually causes ROS accumulation in plants (Xiong et al. 2002; Yang and Guo 2018). A high concentration of ROS leads to oxidative damage to membranes (lipid peroxidation), proteins, RNA, and DNA molecules (Mittler 2002). Several enzymes such as superoxide (SOD), peroxidase (POD), and catalase (CAT) contribute to the ROS detoxification in plants (Mittler et al. 2004). Our transcriptomic analysis showed that many genes, involved in ROS metabolism, were enriched (Fig 2C.). Hence, we investigated if ROS homeostasis was affected in Osflp and OE plants under salt stress conditions. Considering hydroponic cultivation is more stable and easier for stress treatment, we performed the following measurements with hydroponic cultivation. Two-week-old seedlings of ZH11, Osflp, COM, and $\mathrm{OE}$ were treated with or without $150 \mathrm{mM} \mathrm{NaCl}$ for 3 days and 10 days, respectively, afterward following 3 days recovery (Fig. 4A). Salt stress generally increased soluble sugar concentration (Dubey and Singh 1999). Thus we performed measurements with survival rate and the soluble sugar content. It showed that the survival rate and the soluble sugar content were significantly lower in Osflp but higher in OE plants, compared with ZH11 and COM under salt stresses (Fig. 4B-C). Then, we checked the enzyme activities of SOD, POD, and CAT in ZH11, Osflp, OE, and COM. Under non-salt treatment conditions, the activities of these enzymes in Osflp and OE were very similar to those in ZH11 and COM. However, upon salt stresses, the activities of these enzymes significantly reduced in $O s f l p$ but enhanced in $\mathrm{OE}$ (Fig. 4D-F). In addition, with salt treatment conditions, more $\mathrm{H}_{2} \mathrm{O}_{2}$ accumulated in $O s f l p$ but less in OE (Fig. 4G). Consistently, diaminobenzidine (DAB) and nitro blue tetrazolium (NBT) staining also confirmed the higher contents of ROS in Osflp relative to ZH11 and COM (Fig. 4H and I). Collectively, these results suggest that OsFLP affects ROS accumulation in response to salt stress. 
Under salt stress conditions, intracellular $\mathrm{Na}^{+}$and $\mathrm{K}^{+}$homeostasis is essential for cell activities and is crucial for salt tolerance in plants (Zhu 2003; Horie et al. 2001). To test if $\mathrm{Na}^{+}$and $\mathrm{K}^{+}$homeostasis was changed in Osflp mutant and $\mathrm{OE}$ lines with salt stress, we measured $\mathrm{Na}^{+}$and $\mathrm{K}^{+}$contents in both shoots and roots. Under normal growth conditions, no significant differences in $\mathrm{Na}^{+}$and $\mathrm{K}^{+}$contents were observed in both Osflp and OE lines compared to ZH11 and COM. However, with $150 \mathrm{mM} \mathrm{NaCl}$ treatment, $\mathrm{Na}^{+}$contents were significantly higher in both shoots and roots of $O s f l p$ mutant compared to ZH11 and COM. In contrast, in OE lines, $\mathrm{Na}^{+}$contents were much lower in both shoots and roots (Fig. 5A and D). Whereas, $\mathrm{K}^{+}$contents behaved oppositely (Fig. 5B and E). Thus, with $\mathrm{NaCl}$ treatment, the ratio of $\mathrm{Na}^{+} / \mathrm{K}^{+}$in both shoots and roots was increased in Osflp and decreased in OE plants (Fig. 5C and F). This result indicated that the excessive $\mathrm{Na}^{+}$was accumulated in $O s f l p$ under the salt stress condition.

\section{OSCDKB1;1 is the direct target of OsFLP}

335 In Arabidopsis, AtFLP/AtMYB88 directly controlled AtCDKB 1;1 expression to regulate symmetric division in stomatal development (Xie et al. 2010a). OsCDKB1;1 shares $88.5 \%$ amino acid identity with AtCDKB1;1 and AtCDKB1;2 (Qu et al. 2018). To identify the target of OsFLP, OsCDKB1;1 was taken as one potential candidate. The expression of $O s C D K B 1 ; 1$ was significantly induced in $O s f l p$ but repressed in OE, compared with that in ZH11 and COM (Fig. 6A), suggesting that OsFLP negatively

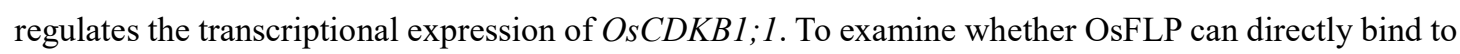
the promoter region of $O S C D K B 1 ; 1$, a yeast one-hybrid assay was conducted. The 2266 bp upstream of the transcriptional start site (ATG) was truncated into several fragments. The results showed that OsFLP is directly bound to the region of 137 bp upstream of ATG, but not to the other regions of OSCDKB1;1

344 promoter (Fig. 6B). To determine the exact sequence which was bound by OsFLP, we scanned the OsCDKB 1; 1 promoter region $137 \mathrm{bp}$ upstream of ATG (-137 bp to -1bp) and found six potential binding sites including $-130 \mathrm{bp}$ to $-126 \mathrm{bp}$ (TTCCC), -125 bp to $-121 \mathrm{bp}$ (TTCCC), -114 bp to $-110 \mathrm{bp}$ (ATCCC), -78 bp to -74 bp (ATCCC), -18 bp to -14 bp (ATCGG) and -10 bp to -6 bp (TTCGG) (Fig. 6C). To confirm the binding specificity, we divided this 137 bp promoter region into three fragments with -137 bp to -95 bp, $-95 \mathrm{bp}$ to $-45 \mathrm{bp}$ and -45 bp to $-1 \mathrm{bp}$, respectively, and performed electrophoretic mobility shift assay (EMSA) using recombinant OsFLP. Ultimately, we found that -125 bp to $-121 \mathrm{bp}$ (TTCCC) and $-78 \mathrm{bp}$ to $-74 \mathrm{bp}$ (ATCCC) were required for OsFLP binding with obviously shifted bands. By 
shifted bands (Fig. 6D-F).

\section{$O S C D K B 1 ; 1$ negatively regulates salt stress responses}

355 As the expression of $O s C D K B 1 ; 1$ was directly regulated by OsFLP (Fig. 6A), we were wondering if OsCDKB1;1 was also involved in the salt stress responses in rice. Then, we were encouraged to examine whether OsCDKB1;1 was required for salt tolerance. We exposed two-week-old seedlings of $O s C D K B 1 ; 1$ over-expression plant (OsCDKB1;1-OE) and the OsCDKB1;1 knockdown plant (OsCDKB1;1-RNAi) (Qu et al. 2018) to $150 \mathrm{mM} \mathrm{NaCl}$ stress conditions, with ZH11 as control. After 12 days of salt stress and 3 days recovery, $O s C D K B 1 ; 1-\mathrm{OE}$ plants exhibited more sensitivity to salt stress, while OsCDKB1;1-RNAi behaved more tolerance to salt stress compared with ZH11 (Fig. 7A). Consistently, under salt stresses, the survival rate was significantly lower in $O S C D K B 1 ; 1-\mathrm{OE}$ plants but much higher in $O s C D K B 1 ; 1$-RNAi plants compared with ZH11 (Fig. 7B). It suggested that $O s C D K B 1 ; 1$ had a negative function on salt stress tolerance in rice.

To investigated whether the ROS accumulation was impacted in $O s C D K B 1 ; 1-$ RNAi plants, we measured the $\mathrm{H}_{2} \mathrm{O}_{2}$ content with or without salt stresses. Intriguingly, upon salt stress, compared with ZH11, a higher level of $\mathrm{H}_{2} \mathrm{O}_{2}$ was accumulated in $O S C D K B$ 1; 1-OE plants, while the lower level of $\mathrm{H}_{2} \mathrm{O}_{2}$ was accumulated in $O S C D K B 1 ; 1$-RNAi plants (Fig. 7C), suggesting that $O S C D K B 1 ; 1$ influenced the content of $\mathrm{H}_{2} \mathrm{O}_{2}$ under salt stress condition. Collectively, these results indicate that $O s C D K B 1 ; 1$ plays a negative role in response to salt stress by increasing ROS production.

\section{Discussion}

It has been implicated that $A t F L P$ as a multiple functional regulator involves several aspects of plant physiological processes, including stomatal development, abiotic stress response, root gravitropism, and female reproductive development (Xie et al. 2010a; Xie et al. 2010b; Wang et al. 2015; Makkena et al. 2012). In rice, it has been reported that OsFLP controls the orientation of the GMC symmetric division in stomatal development (Wu et al. 2019), but the downstream targets are unknown (Lai et al. 2005). It seems that the function of FLP is conserved and divergent in monocots and dicots plants. However, the function of OsFLP in rice is still largely unknown. In this study, by transcriptomic analysis, the role of OsFLP was involved in many aspects, while we mainly focused on the salt stress responses. We also found that the Osflp mutant was sensitive to salt stress with more ROS accumulation and the OE lines behaved conversely. It indicates that OsFLP acts as a key regulator in salt tolerance in rice. 

salt stress conditions, the uptake of $\mathrm{Na}^{+}$competes with $\mathrm{K}^{+}$and even the salt stress blocks the $\mathrm{K}^{+}$specific transporters (Zhu 2003). Our findings showed that more $\mathrm{Na}^{+}$was taken up into the cells of $O s f l p$ under salt stress conditions. In this process, some sodium transporters may be involved (Fig. 7D). Interestingly, the integrative analysis of RNA-seq and ChIP-seq identified the $\mathrm{Ca}^{2+} / \mathrm{Na}^{+}$antiporter (Os03g0411300) with increased expression, and the potassium channel (Os01g0846300) with decreased expression (Supplementary Table S2). Hence, we proposed that OsFLP may regulate the activity of $\mathrm{Ca}^{2+} / \mathrm{Na}^{+}$ antiporter and potassium channels to control the intracellular concentration of $\mathrm{Na}^{+}$. In Arabidopsis, AtFLP together with $A t C D K B 1 ; 1$ and $A t C D K B 1 ; 2$ controls the stomatal division. However, in rice, no obvious phenotype in stomatal development in $O s c d k b 1 ; 1$ was found. OsCDKB1;1 may have functional redundancy with $O s C D K B 1 ; 2$ in regulating the stomatal development (Qu et al. 2018; Menges et al. 2005; Porceddu et al. 2001; Andersen et al. 2008). However, in Arabidopsis, the response to salt stress involves a rapid decline of cell cycle activity (Burssens et al. 2000; West et al. 2004), and the expression of CDKB1;1 and CDKB1;2 was downregulated under salt stress (Mahapatra and Roy 2021). Intriguingly, in our findings, Oscdkb1;1 exhibited significantly salt tolerance with less ROS accumulation under salt stress conditions, suggesting that $O_{s} C D K B 1 ; 1$ had a negative function in salt tolerance. Considering OsFLP directly binding to the promoter region of $O S C D K B 1 ; 1$ and repressing its transcription, we conclude that OsFLP associated with $O S C D K B 1 ; 1$ confers salt tolerance in rice. Thus, our findings uncover a novel OsFLP-OSCDKB1;1 module conferring salt tolerance by involving ROS accumulation in rice.

In addition, by RNA-seq analysis, many PRXs genes were identified in Osflp. PRXs mainly function in catalyzing $\mathrm{H}_{2} \mathrm{O}_{2}$ scavenging and ROS production (Bhatt and Tripathi 2011; Almagro et al. 2009), indicating that OsFLP might directly or indirectly regulate PRXs to influence the ROS contents in plants. Hence, in the future, it will be interesting to explore how OsFLP mediates the transcription of PRXs to affect the ROS accumulation and will be beneficial for uncovering the mechanism of OsFLP involved in physical processes of salt tolerance.

Based on our findings, we proposed a working model for OsFLP-OsCDKB 1;1 mediated salt tolerance in rice (Fig. 7D). Under salt stress conditions, in wild-type plant ZH11, OsFLP protein binds to the promoter region of $O s C D K B 1 ; 1$ and represses the expression of $O_{S} C D K B 1 ; 1$ leading to a decrease in 
the ROS accumulation resulting in salt tolerance in rice. In $O s f l p$ mutant plant, the expression of

OsCDKB1;1 is activated and the ROS accumulation is increased, ultimately, enhancing salt sensitivity

in rice. Together, our findings extend the knowledge about the role of OsFLP in rice, especially in salt stress response, indicating the potential benefits for salt-tolerant rice cultivars' improvement.

\section{Accession numbers}

Sequences used in this study were download from the Rice Genome Annotation Project Database (http://rice.plantbiology.msu.edu/) with the following accession numbers: OsFLP LOC_Os07g43420;

OsCDKB1;1 LOC_Os01g67160; OsPRX107 LOC_Os07g44550; OsPRX109 LOC_Os07g47990;

OsPRX129 LOC_Os10g41720; OsPRX112 LOC_Os07g48030; OsRab16A LOC_Os02g04680;

OsNAC5 LOC_Os07g37920; OsDREB2A LOC_Os01g07120; OsDREB1F LOC_Os01g73770;

OsACTIN2 LOC_Os03g50885.

\section{Acknowledgements}

This work was supported by grants from the Strategic Priority Research Program of the Chinese Academy of Sciences (grant number. XDA24010303) and the National Natural Science Foundation of

China (grant numbers 31970804 and 32170722 to J. L, grant number 31470362 to J.-J. Z and grant number 31871377 and 32070723 to K.-Z. Y).

\section{References}

Almagro L, Gómez Ros LV, Belchi-Navarro S, Bru R, Ros Barceló A, Pedreño MA (2009) Class III peroxidases in plant defence reactions. J Exp Bot 60: 377-390. https://10.1093/jxb/ern277 Requirement of B2-type cyclin-dependent kinases for meristem integrity in Arabidopsis thaliana. Plant Cell 20: 88-100. https://10.1105/tpc.107.054676 Bhatt I, Tripathi BN (2011) Plant peroxiredoxins: catalytic mechanisms, functional significance and future perspectives. Biotechnol Adv 29: 850-859. https://10.1016/j.biotechadv.2011.07.002 Boudolf V, Rombauts S, Naudts M, Inzé D, De Veylder L (2001) Identification of novel cyclin-dependent kinases interacting with the CKS1 protein of Arabidopsis. J Exp Bot 52: 1381-1382. https://doi.org/10.1093/jexbot/52.359.1381 Expression of cell cycle regulatory genes and morphological alterations in response to salt stress in Arabidopsis thaliana. Planta 211: 632-640. https://10.1007/s004250000334

De Veylder L, Joubès J, Inzé D (2003) Plant cell cycle transitions. Curr Opin Plant Biol 6: 536-543. https://10.1016/j.pbi.2003.09.001

Dewitte W, Murray JA (2003) The plant cell cycle. Annu Rev Plant Biol 54: 235-264. https://10.1146/annurev.arplant.54.031902.134836 
Dubey RS, Singh AK (1999) Salinity induces accumulation of soluble sugars and alters the activity of sugar metabolizing enzymes in rice plants. Biol Plant 42:233-9. https://doi.org/10.1023/A:1002160618700

El-Kereamy A, Bi YM, Ranathunge K, Beatty PH, Good AG, Rothstein SJ (2012) The rice R2R3-MYB transcription factor OsMYB55 is involved in the tolerance to high temperature and modulates amino acid metabolism. PLoS One 7: e52030. https://10.1371/journal.pone.0052030

Gray JE, Holroyd GH, van der Lee FM, Bahrami AR, Sijmons PC, Woodward FI, Schuch W, Hetherington AM (2000) The HIC signalling pathway links CO2 perception to stomatal development. Nature 408: 713-716. https://10.1038/35047071

Gupta B, Huang B (2014) Mechanism of salinity tolerance in plants: physiological, biochemical, and molecular characterization. Int J Genomics 2014: 701596. https://10.1155/2014/701596

Hiei Y, Ohta S, Komari T, Kumashiro T (1994) Efficient transformation of rice (Oryza sativa L.) mediated by Agrobacterium and sequence analysis of the boundaries of the T-DNA. Plant J 6: 271-282. https://10.1046/j.1365-313x.1994.6020271.x

Horie T, Yoshida K, Nakayama H, Yamada K, Oiki S, Shinmyo A (2001) Two types of HKT transporters with different properties of $\mathrm{Na}+$ and $\mathrm{K}+$ transport in Oryza sativa. Plant J 27: 129-138. https://10.1046/j.1365-313x.2001.01077.x

James RA, Blake C, Byrt CS, Munns R (2011) Major genes for Na+ exclusion, Nax1 and Nax2 (wheat HKT1;4 and HKT1;5), decrease Na+ accumulation in bread wheat leaves under saline and waterlogged conditions. J Exp Bot 62: 2939-2947. https://10.1093/jxb/err003

Kanaoka MM, Pillitteri LJ, Fujii H, Yoshida Y, Bogenschutz NL, Takabayashi J, Zhu JK, Torii KU (2008) SCREAM/ICE1 and SCREAM2 specify three cell-state transitional steps leading to arabidopsis stomatal differentiation. Plant Cell 20: 1775-1785. https://10.1105/tpc.108.060848

Kreps JA, Wu Y, Chang HS, Zhu T, Wang X, Harper JF (2002) Transcriptome changes for Arabidopsis in response to salt, osmotic, and cold stress. Plant Physiol 130: 2129-2141. https://10.1104/pp.008532 Lai LB, Nadeau JA, Lucas J, Lee EK, Nakagawa T, Zhao L, Geisler M, Sack FD (2005) The Arabidopsis R2R3 MYB proteins FOUR LIPS and MYB88 restrict divisions late in the stomatal cell lineage. Plant Cell 17: 2754-2767. https://10.1105/tpc.105.034116

Lei Q, Lee E, Keerthisinghe S, Lai L, Li M, Lucas JR, Wen X, Ren X, Sack FD (2015) The FOUR LIPS and MYB88 transcription factor genes are widely expressed in Arabidopsis thaliana during development. Am J Bot 102: 1521-1528. https://10.3732/ajb.1500056

Lv Y, Yang M, Hu D, Yang Z, Ma S, Li X, Xiong L (2017) The OsMYB30 Transcription Factor Suppresses Cold Tolerance by Interacting with a JAZ Protein and Suppressing $\beta$-Amylase Expression. Plant Physiol 173: 1475-1491. https://10.1104/pp.16.01725

Mahapatra K, Roy S (2021) SOG1 transcription factor promotes the onset of endoreduplication under salinity stress in Arabidopsis. Sci Rep 11: 11659. https://10.1038/s41598-021-91293-1

Mäkelä P, Kontturi M, Pehu E, Somersalo S (1999) Photosynthetic response of drought- and salt-stressed tomato and turnip rape plants to foliar-applied glycinebetaine. 105: 45-50. https://https://doi.org/10.1034/j.1399-3054.1999.105108.x

Makkena S, Lee E, Sack FD, Lamb RS (2012) The R2R3 MYB transcription factors FOUR LIPS and MYB88 regulate female reproductive development. J Exp Bot 63: 5545-5558. https://10.1093/jxb/ers209 Martin C, Paz-Ares J (1997) MYB transcription factors in plants. Trends Genet 13: 67-73. https://10.1016/s0168-9525(96)10049-4

Matsui A, Ishida J, Morosawa T, Mochizuki Y, Kaminuma E, Endo TA, Okamoto M, Nambara E, 

Arabidopsis transcriptome analysis under drought, cold, high-salinity and ABA treatment conditions using a tiling array. Plant Cell Physiol 49: 1135-1149. https://10.1093/pcp/pcn101

Menges M, de Jager SM, Gruissem W, Murray JA (2005) Global analysis of the core cell cycle regulators of Arabidopsis identifies novel genes, reveals multiple and highly specific profiles of expression and provides a coherent model for plant cell cycle control. Plant J 41: 546-566. https://10.1111/j.1365313X.2004.02319.x

Miao BH, Han XG, Zhang WH (2010) The ameliorative effect of silicon on soybean seedlings grown in potassium-deficient medium. Ann Bot 105: 967-973. https://10.1093/aob/mcq063

Miller G, Suzuki N, Ciftci-Yilmaz S, Mittler R (2010) Reactive oxygen species homeostasis and signalling during drought and salinity stresses. Plant Cell Environ 33: 453-467. https://10.1111/j.13653040.2009.02041.x

Mittler R (2002) Oxidative stress, antioxidants and stress tolerance. Trends Plant Sci 7: 405-410. https://10.1016/s1360-1385(02)02312-9

Mittler R, Vanderauwera S, Gollery M, Van Breusegem F (2004) Reactive oxygen gene network of plants. Trends Plant Sci 9: 490-498. https://10.1016/j.tplants.2004.08.009

Noctor G, Foyer CH (1998) ASCORBATE AND GLUTATHIONE: Keeping Active Oxygen Under Control. Annu Rev Plant Physiol Plant Mol Biol 49: 249-279. https://10.1146/annurev.arplant.49.1.249 Ponce KS, Meng L, Guo L, Leng Y, Ye G (2021) Advances in Sensing, Response and Regulation Mechanism of Salt Tolerance in Rice. Int J Mol Sci 22. https://10.3390/ijms22052254

Porceddu A, Stals H, Reichheld JP, Segers G, De Veylder L, Barroco RP, Casteels P, Van Montagu M, Inzé D, Mironov V (2001) A plant-specific cyclin-dependent kinase is involved in the control of G2/M progression in plants. J Biol Chem 276: 36354-36360. https://10.1074/jbc.M011060200

Qu X, Yan M, Zou J, Jiang M, Yang K, Le J (2018) A2-type cyclin is required for the asymmetric entry division in rice stomatal development. J Exp Bot 69: 3587-3599. https://10.1093/jxb/ery158

Roy SJ, Negrão S, Tester M (2014) Salt resistant crop plants. Curr Opin Biotechnol 26: 115-124. https://10.1016/j.copbio.2013.12.004

Shen H, Zhong X, Zhao F, Wang Y, Yan B, Li Q, Chen G, Mao B, Wang J, Li Y, Xiao G, He Y, Xiao H, Li J, He Z (2015a) Overexpression of receptor-like kinase ERECTA improves thermotolerance in rice and tomato. Nat Biotechnol 33: 996-1003. https://10.1038/nbt.3321

Shen Y, Shen L, Shen Z, Jing W, Ge H, Zhao J, Zhang W (2015b) The potassium transporter OsHAK21 functions in the maintenance of ion homeostasis and tolerance to salt stress in rice. Plant Cell Environ 38: 2766-2779. https://10.1111/pce.12586

Shpak ED, McAbee JM, Pillitteri LJ, Torii KU (2005) Stomatal patterning and differentiation by synergistic interactions of receptor kinases. Science 309: 290-293. https://10.1126/science.1109710 Song SY, Chen Y, Chen J, Dai XY, Zhang WH (2011) Physiological mechanisms underlying OsNAC5dependent tolerance of rice plants to abiotic stress. Planta 234: 331-345. https://10.1007/s00425-0111403-2

Tang L, Cai H, Ji W, Luo X, Wang Z, Wu J, Wang X, Cui L, Wang Y, Zhu Y, Bai X (2013) Overexpression of GsZFP1 enhances salt and drought tolerance in transgenic alfalfa (Medicago sativa L.). Plant Physiol Biochem 71: 22-30. https://10.1016/j.plaphy.2013.06.024

Vandepoele K, Raes J, De Veylder L, Rouzé P, Rombauts S, Inzé D (2002) Genome-wide analysis of core cell cycle genes in Arabidopsis. Plant Cell 14: 903-916. https://10.1105/tpc.010445

Wang HZ, Yang KZ, Zou JJ, Zhu LL, Xie ZD, Morita MT, Tasaka M, Friml J, Grotewold E, Beeckman 
T, Vanneste S, Sack F, Le J (2015) Transcriptional regulation of PIN genes by FOUR LIPS and MYB88 during Arabidopsis root gravitropism. Nat Commun 6: 8822. https://10.1038/ncomms9822

West G, Inzé D, Beemster GT (2004) Cell cycle modulation in the response of the primary root of Arabidopsis to salt stress. Plant Physiol 135: 1050-1058. https://10.1104/pp.104.040022

Wu Z, Chen L, Yu Q, Zhou W, Gou X, Li J, Hou S (2019) Multiple transcriptional factors control stomata development in rice. New Phytol 223: 220-232. https://10.1111/nph.15766

Xia XJ, Gao CJ, Song LX, Zhou YH, Shi K, Yu JQ (2014) Role of H2O2 dynamics in brassinosteroidinduced stomatal closure and opening in Solanum lycopersicum. Plant Cell Environ 37: 2036-2050. https://10.1111/pce.12275

Xie Z, Lee E, Lucas JR, Morohashi K, Li D, Murray JA, Sack FD, Grotewold E (2010a) Regulation of cell proliferation in the stomatal lineage by the Arabidopsis MYB FOUR LIPS via direct targeting of core cell cycle genes. Plant Cell 22: 2306-2321. https://10.1105/tpc.110.074609

Xie Z, Li D, Wang L, Sack FD, Grotewold E (2010b) Role of the stomatal development regulators FLP/MYB88 in abiotic stress responses. Plant J 64: 731-739. https://10.1111/j.1365-313X.2010.04364.x Xiong L, Schumaker KS, Zhu JK (2002) Cell signaling during cold, drought, and salt stress. Plant Cell 14 Suppl: S165-183. https://10.1105/tpc.000596

Yang A, Dai X, Zhang WH (2012) A R2R3-type MYB gene, OsMYB2, is involved in salt, cold, and dehydration tolerance in rice. J Exp Bot 63: 2541-2556. https://10.1093/jxb/err431

Yang K, Zhu L, Wang H, Jiang M, Xiao C, Hu X, Vanneste S, Dong J, Le J (2019) A conserved but plantspecific CDK-mediated regulation of DNA replication protein A2 in the precise control of stomatal terminal division. Proc Natl Acad Sci U S A 116: 18126-18131. https://10.1073/pnas. 1819345116

Yang M, Sack FD (1995) The too many mouths and four lips mutations affect stomatal production in Arabidopsis. Plant Cell 7: 2227-2239. https://10.1105/tpc.7.12.2227

Yang Y, Guo Y (2018) Unraveling salt stress signaling in plants. J Integr Plant Biol 60: 796-804. https://10.1111/jipb.12689

Ye Y, Liu B, Zhao M, Wu K, Cheng W, Chen X, Liu Q, Liu Z, Fu X, Wu Y (2015) CEF1/OsMYB103L is involved in GA-mediated regulation of secondary wall biosynthesis in rice. Plant Mol Biol 89: 385401. https://10.1007/s11103-015-0376-0

Zhang H, Liu Y, Wen F, Yao D, Wang L, Guo J, Ni L, Zhang A, Tan M, Jiang M (2014) A novel rice C2H2-type zinc finger protein, ZFP36, is a key player involved in abscisic acid-induced antioxidant defence and oxidative stress tolerance in rice. J Exp Bot 65: 5795-5809. https://10.1093/jxb/eru313 Zhu JK (2003) Regulation of ion homeostasis under salt stress. Curr Opin Plant Biol 6: 441-445. https://10.1016/s1369-5266(03)00085-2

\section{Statements and Declarations}

\section{Funding}

This work was supported by grants from the Strategic Priority Research Program of the Chinese Academy of Sciences (grant number. XDA24010303) and the National Natural Science Foundation of China (grant numbers 31970804 and 32170722 to J. L, grant number 31470362 to J.-J. Z and grant number 31871377 and 32070723 to K.-Z. Y).

\section{Competing Interests}


576 The authors have no relevant financial or non-financial interests to disclose.

\section{Author Contributions}

578 C.-X. Z and J. Z performed the experiments and analyzed the data. H.-C. L, X.-X. Q, J.-X. W, Q.-X.-M.

$579 \mathrm{H}$ and J.-J. Z participated in parts of experiments and data analysis. J. L, K.-Z. Y, C.-X. Z and J. Z

580 designed the experiments. C.-X. Z and J.L wrote the manuscript. All authors read and approved the final

581 manuscript.

582 Data Availability

583 All data generated during this study are included in this published article and its supplementary 584 information files. The RNA-seq datasets generated during the current study are available in the NCBI

585 Sequence Read Archive repository,

586 https://dataview.ncbi.nlm.nih.gov/object/PRJNA805061?reviewer=m847fdbhc5h8hlajg783fodski

587 https://ataview.ncbi.nlm.nih.gov/object/PRJNA805087?reviewer=gj1 raljgoss270nmmf2d8dgmfu

588 The ChIP-seq datasets generated during the current study are available in the NCBI Sequence Read

589 Archive (SRA) repository,

590 https://dataview.ncbi.nlm.nih.gov/object/PRJNA803610?reviewer=8ark21 mh1c3mrblaoj1 fnpek34

\section{$591 \quad$ Figure captions}

592 Fig. 1 The characterization of Osflp

593 A. Osflp mutant line was generated by TILLING system. A point mutation nucleotide G to A was 594 identified in the sixth exon at position 2126 bp downstream of ATG which led to the substitution of amino 595 acid Ala with Thr

596 B. Multiple alignments of amino acid sequences of OsFLP, AtFLP, AtMYB88, Bradi1g20586, 597 Glyma01g05190, Solyc04g005710, Solyc05g007160, Zm24064630 showed the mutation site of Osflp 598 located in the highly conserved region of R3 domain

599 C-E. Normal stomata in ZH11 and complementation line. Abnormal division and differentiation stomata 600 in Osflp was indicated with red arrowhead, Scale bars $=20 \mu \mathrm{m}$

601 Fig. 2 Identification of potential targets of OsFLP by transcriptomic analysis

602 A. Volcano Plot shows the up-and down-regulated genes in Osflp mutant compared with ZH11

603 B. The potential proteins or biological processes which are related to OsFLP, are identified by RNA-seq 604 analysis 

analysis

607 D. The integrative of RNA-seq and ChIP-seq. In total, 125 genes were identified as the potential targets of OsFLP. Among them, 71 genes were induced and 54 genes were repressed E-H. The expression of salt stress-related genes OsRab16A, OsNAC5, OsDREB2A, OsDREB1F were detected using RT-qPCR with or without salt treatment in ZH11, Osflp, COM and OE lines, respectively. OsActin was always taken as the reference gene. Data are means $\pm \mathrm{SD}$. The student's $t$-test was performed. Statistically significant differences are indicated with asterisks: ${ }^{*}, P<0.05,{ }^{* *}, P<0.01$ Fig. 3 Osflp mutants are more sensitive to salt stress

A. Salt stress tolerance phenotype assay of ZH11, Osflp, COM and OsFLP overexpression line (OE) seedlings with or without $\mathrm{NaCl}$ treatment. Seedlings were grown on 1/2 MS plates for three days and transferred to $1 / 2 \mathrm{MS}$ plates supplemented with or without $200 \mathrm{mM} \mathrm{NaCl}$ for 5 days. Scale bars $=1 \mathrm{~cm}$ B and C. The root length and the shoot length of ZH11, Osflp, COM and OE seedlings were quantified, respectively, in D. Data are means $\pm \mathrm{SD}$. The student's $t$-test was performed. Statistically significant differences are indicated with asterisks: ${ }^{* *}, P<0.01$ D. Salt stress tolerance phenotype assay of ZH11, Osflp, COM, and OE seedlings were grown on soil. 7day-old seedlings cultivated on soil irrigated with water or water supplement with $200 \mathrm{mM} \mathrm{NaCl}$ for 15 days and 21 days, respectively. Scale bars $=10 \mathrm{~cm}$ E. Survival rates of ZH11, Osflp, COM, and OE plants after 21 days of stress treatment were analyzed. Data are means \pm SD. The student's t-test was performed. Statistically, significant differences are indicated with asterisks: **, $P<0.01$

Fig. 4 More ROS was accumulated in Osflp rice mutant under salt stress conditions

A. Salt stress phenotype assay of ZH11, Osflp, COM, and OE seedlings cultivated with hydroponic culture. Two-week-old seedlings were treated with or without $150 \mathrm{mM} \mathrm{NaCl}$ in hydroponic culture after 3 days, 10 days, and 3 days of restored growth.

630 B. Survival rate was analyzed after 3 days of recovery.

631 C. Soluble sugar content was measured after 3 days of recovery.

632 D-G. SOD activity (D), POD activity (E), CAT activity (F), and $\mathrm{H}_{2} \mathrm{O}_{2}$ content (G) were assayed after 3 
Student's $t$ - test was performed. Statistically significant differences are indicated with asterisks: *, $P<0.05, * *, P<0.01$

H-I. Both DAB (H) and NBT(I) staining showed more ROS accumulation in Osflp mutant leaves treated with $150 \mathrm{mM} \mathrm{NaCl}$ treatment after 24 hours. Scale bars $=0.5 \mathrm{~cm}$

638 Fig. 5 Osflp mutant accumulates more $\mathrm{Na}^{+}$and less $\mathrm{K}^{+}$in shoots and roots under salt stress

$639 \mathrm{Na}^{+}$and $\mathrm{K}^{+}$contents assay in root and shoot of ZH11, Osflp, COM and OE lines of rice plants. Two640 week-old seedlings were treated with or without $150 \mathrm{mM} \mathrm{NaCl}$ for 4 days

641 A. and B. $\mathrm{Na}^{+}$and $\mathrm{K}^{+}$contents were measured in the shoot

642 C. The ratio of $\mathrm{Na}^{+}$to $\mathrm{K}^{+}$in the shoot was calculated according to the measurements in $\mathrm{A}$. and B

643 D. and E. $\mathrm{Na}^{+}$and $\mathrm{K}^{+}$contents were measured in root

644 F. The ratio of $\mathrm{Na}^{+}$to $\mathrm{K}^{+}$in root was calculated according to the measurements in D. and E. Data are 645 means \pm SD from three biological replicates. The student's t-test was performed. Statistically, significant 646 differences were indicated with asterisks: *, $\mathrm{p}<0.05,{ }^{* *}, \mathrm{p}<0.01$

647 Fig. 6 OsFLP directly binds to the promoter region of $O s C D K B 1: 1$ and regulates its transcriptional 648 expression

649 A. The transcriptional expression of $\operatorname{Os} C D K B 1 ; 1$ was negatively regulated by OsFLP. The transcriptional 650 level of OsCDKB1;1 was detected by RT-qPCR in ZH11, Osflp, COM, and OE lines of rice plants

651 B. OsFLP binds to the promoter region of $O s C D K B 1 ; 1$ in yeast one-hybrid assay. The full length of the 652 promoter region of $O s C D K B 1 ; 1$ was truncated into nine fragments. The area from -137 bp to -1 bp was 653 identified as the minimal promoter region, which is the region for the binding of OsFLP

654 C. Schematic diagram of OsCDKB 1;1 promoter region showing potential binding sites of OsFLP in three 655 different regions including P1, P2, and P3

656 D-F. Electrophoretic mobility shift assays (EMSA) indicated OsFLP specifically bound to the promoter 657 region of the $O s C D K B 1 ; 1$. The arrows indicate the protein-DNA complexes. The asterisks indicate the 658 non-specific binding. The competitor was the probe without biotin label

659 Fig. 7 OsCDKB 1;1 negatively regulates the response to salt stress in rice

660 A. Salt stress phenotype analysis of ZH11, OsCDKB1;1-OE, OsCDKB1;1-RNAi. Two-week-old 661 seedlings were cultivated in hydroponic culture with or without $150 \mathrm{mM} \mathrm{NaCl}$ for 12 days and then 662 restored growth for 3 days 

recovery

C. The content of $\mathrm{H}_{2} \mathrm{O}_{2}$ was monitored in ZH11, OsCDKB1;1-OE, OsCDKB1;1-RNAi after salt stress recovery. Data in B-E are means $\pm \mathrm{SD}$ from three biological replicates. The student's $t$-test was performed. Statistically, significant differences are indicated with asterisks: ${ }^{* *}, P<0.01$

D. Proposed working model for OsFLP-OSCDKB1;1 transcriptional regulation module mediated salt stress response in rice. Under salt stress conditions, in ZH11, OsFLP binds to the promoter region of OsCDKB1;1 to repress the transcriptional expression of $O s C D K B 1 ; 1$ resulting in less ROS accumulation, ultimately conferring salt tolerance (in the left panel). However, in Osflp mutant plants, the expression of $O S C D K B 1 ; 1$ is enhanced, and promotes the ROS accumulation in plants, thereby leading to salt sensitivity in rice (in the right panel). OsFLP $\mathrm{m}_{\mathrm{m}}$ indicates mutation of OsFLP

\section{Supplementary Information}

Fig. S1 Phylogenetic tree shows the relationship of FLP proteins from algae, lower plants and higher 676 plants

677 Fig. S2 RT-qPCR was performed to verify the results of the RNA-seq

678 Fig. S3 KEGG enrichment analysis with ChIP-seq

679 Fig. S4 KEGG enrichment analysis with genes identified by the integrative analysis of RNA-seq data and ChIP-seq data

$681 \quad$ Fig. S5 The expression of $O S F L P$ upon salt stress

682 Fig. S6 Osflp mutants are more sensitive to salt stress

683 Table. S1 Genes are related to ROS metabolism enriched by RNA-seq analysis

684 Table. S2 Genes are related to salt stress enriched by the integrative analysis of RNA-seq and ChIP-seq

685 Table. S3 Primers were used in this study

686 Dataset. S1 RNA-seq Osflp_vs_ZH11_annotation

687 Dataset. S2 RNA-seq data analysis with categories

688 Dataset. S3 GO analysis with RNA-seq data

689 Dataset. S4 ChIP-seq original data_annotation

690 Dataset. S5 KEGG Enrichment with ChIP-seq data

691 Dataset. S6 RNA-seq and ChIP-seq integrative analysis annotation_Total 
692 Dataset. S7 KEGG Enrichment genes from RNA-seq and ChIP-seq 
Fig. 1

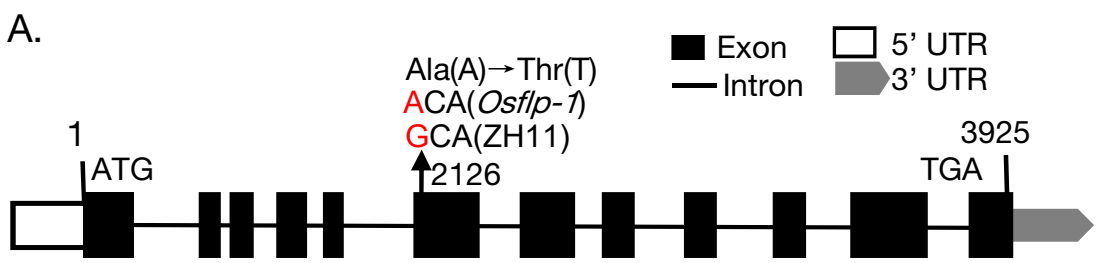

B.

OsFLP

A.MYBse

Brad11920586

31y=201905190

solyco4g005710

$z=26064630$

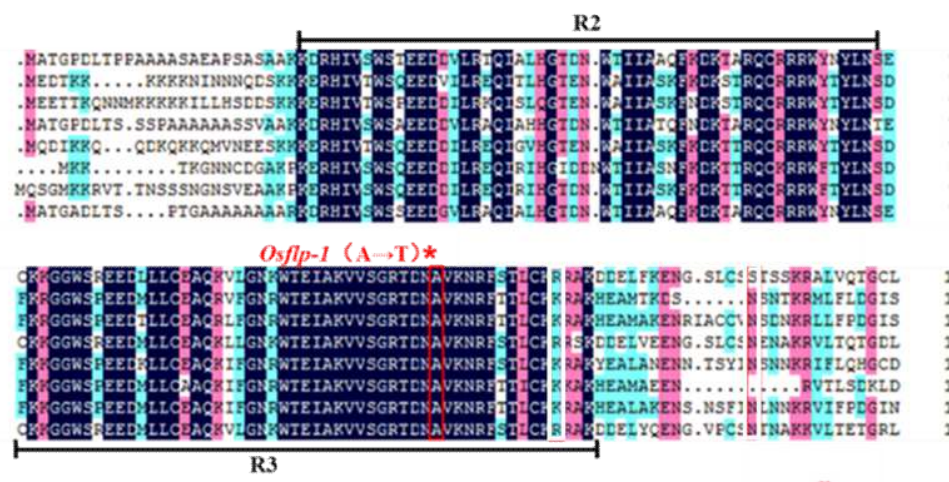

C.

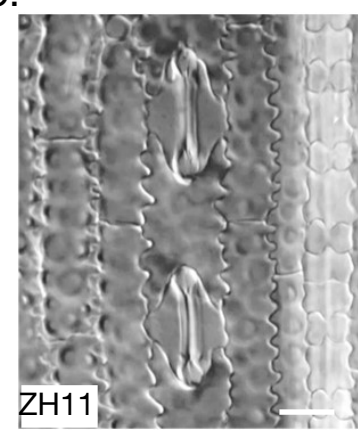

D.

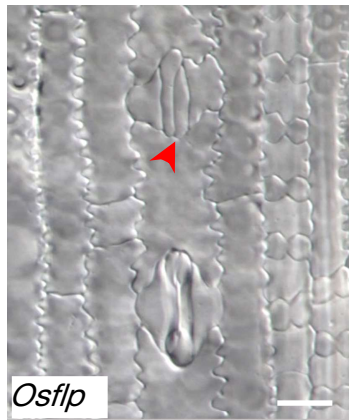

E.

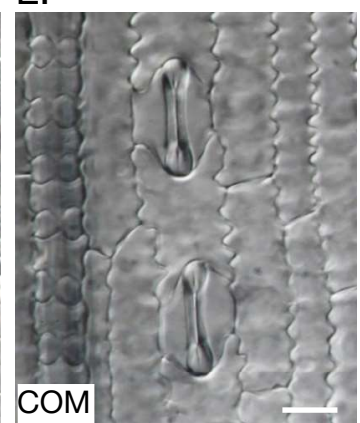


Fig. 2

A

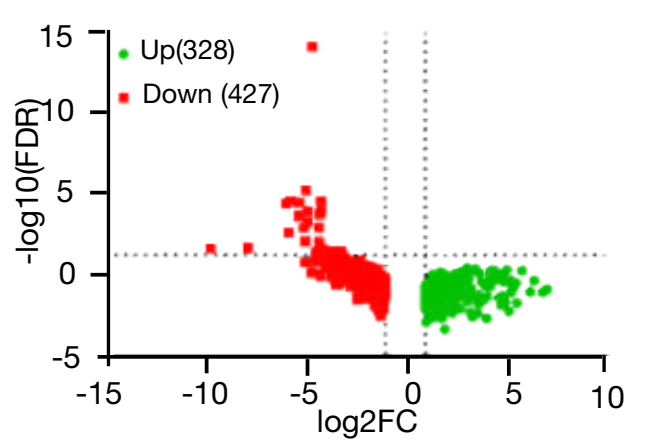

C

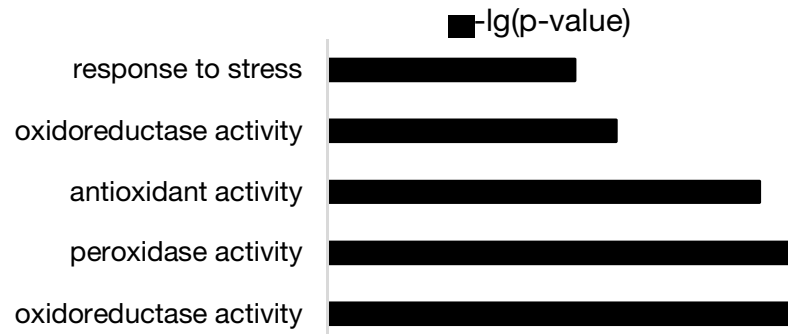

secondary metabolic process

response to oxidative stress

response to stimulus

E
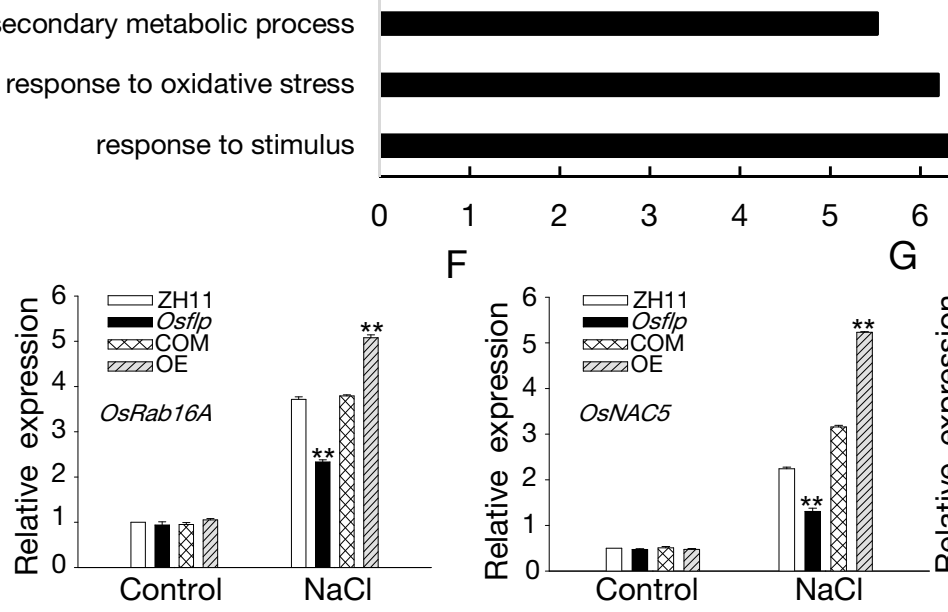

$\mathrm{F}$

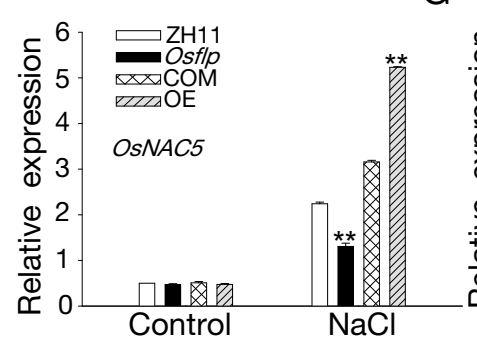

B
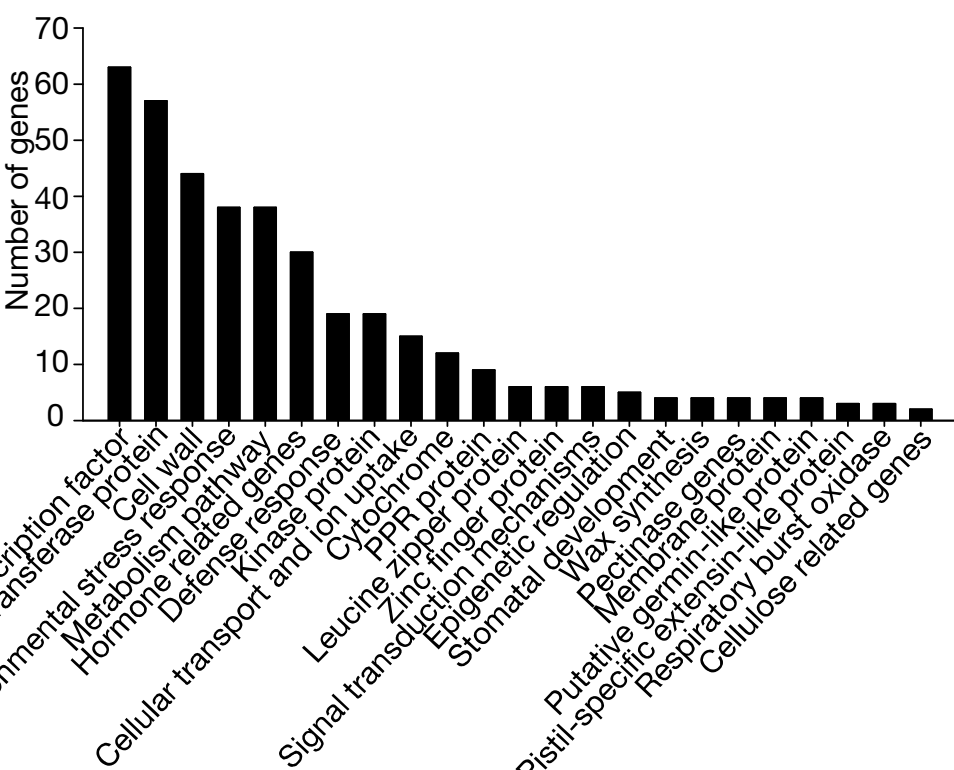

D

OsFLP-associated genes with ChIP-peaks(12899) OsFLP-regulated genes (755)
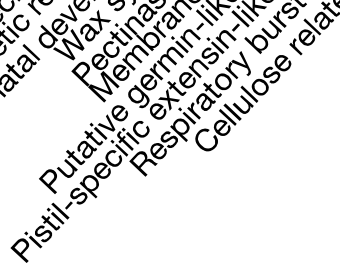

pisero

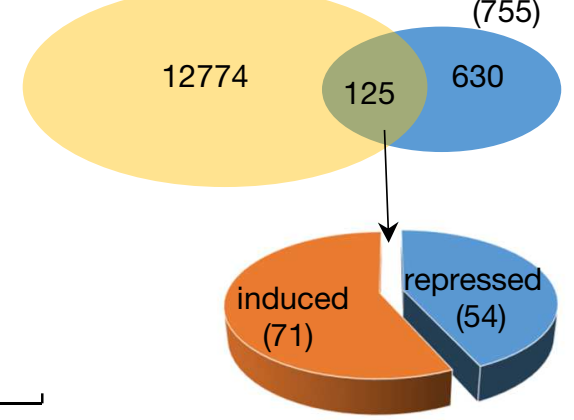

- $\mathrm{H}$
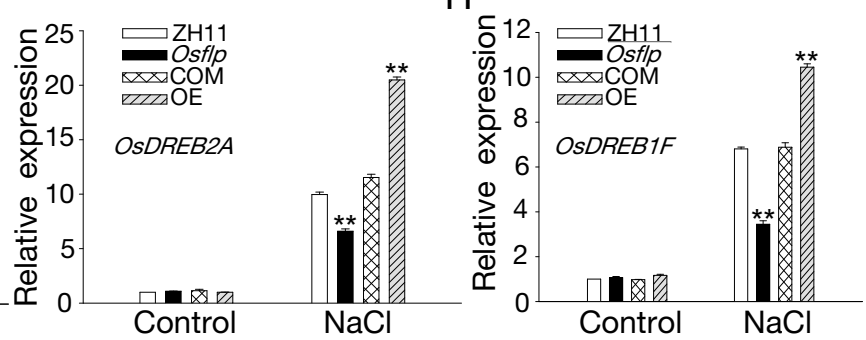
Fig. 3

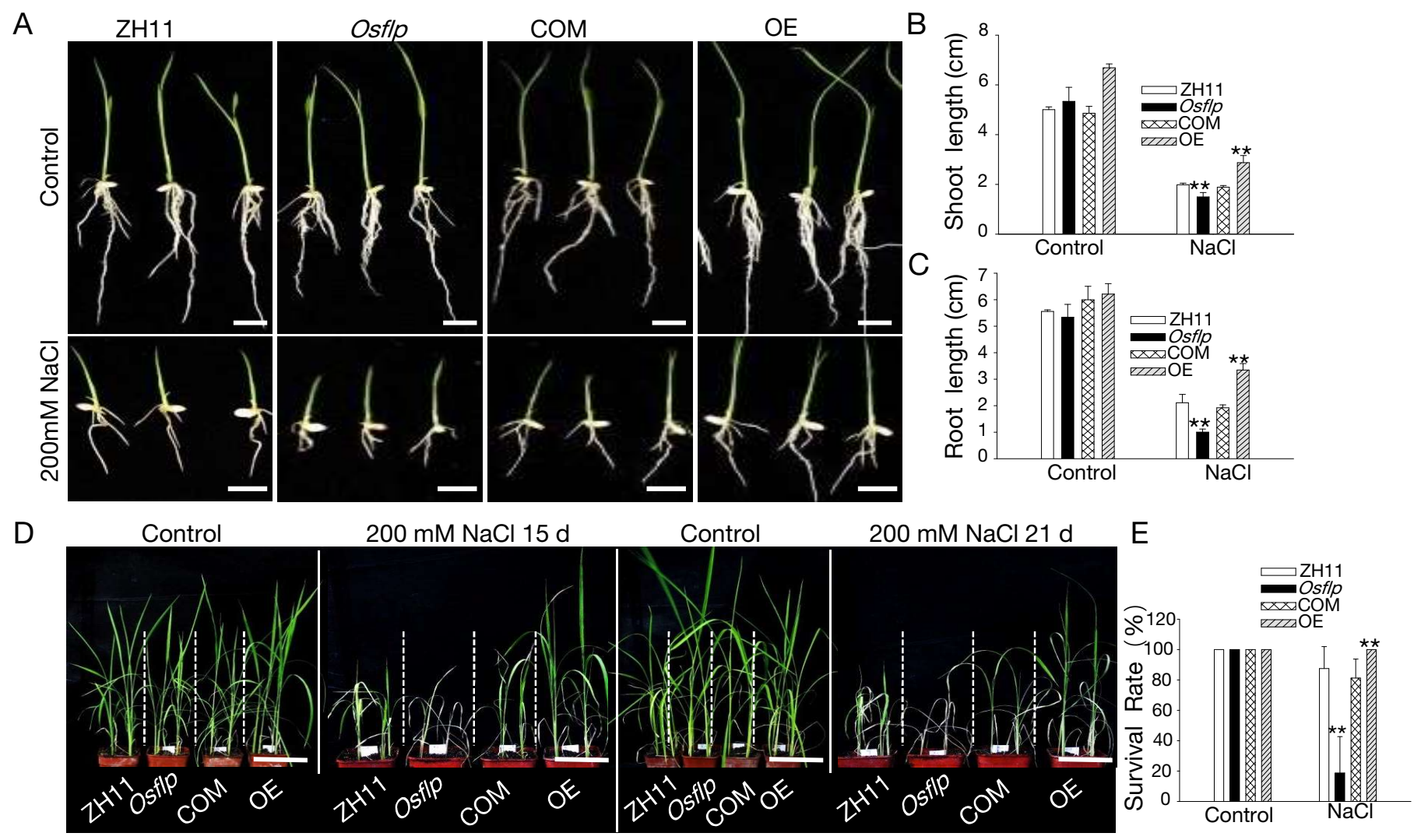


Fig. 4

A
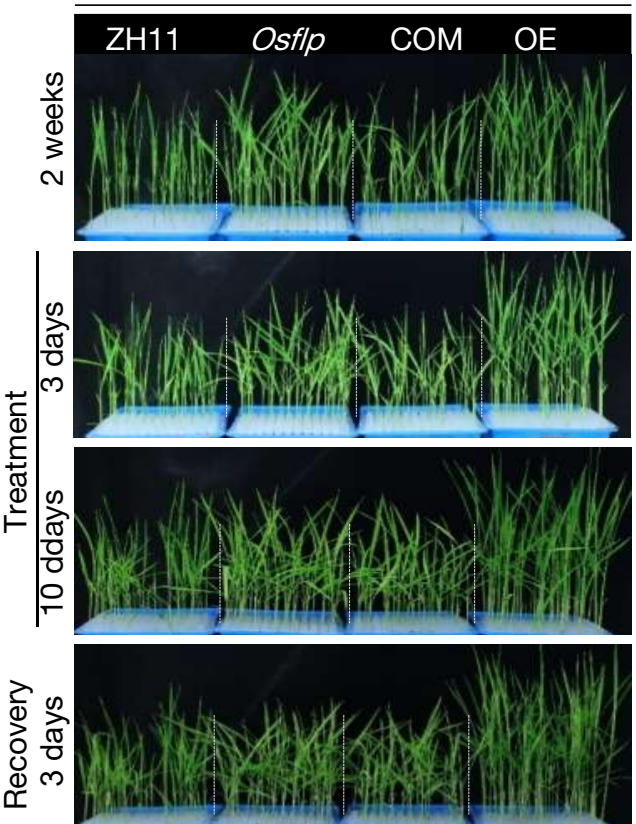

D

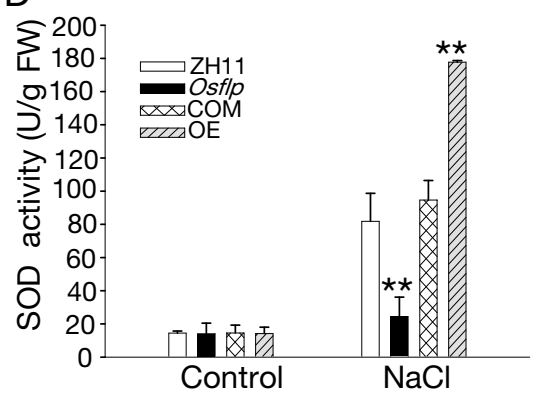

G

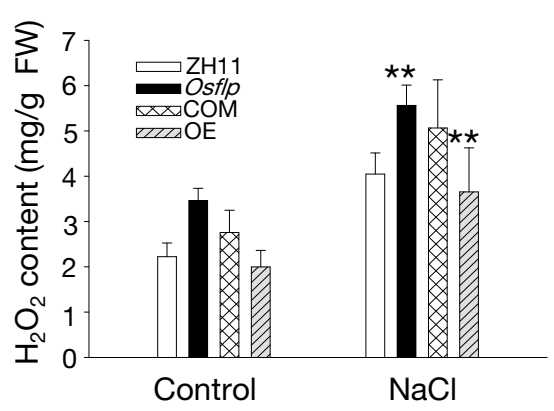

$150 \mathrm{mM} \mathrm{NaCl}$
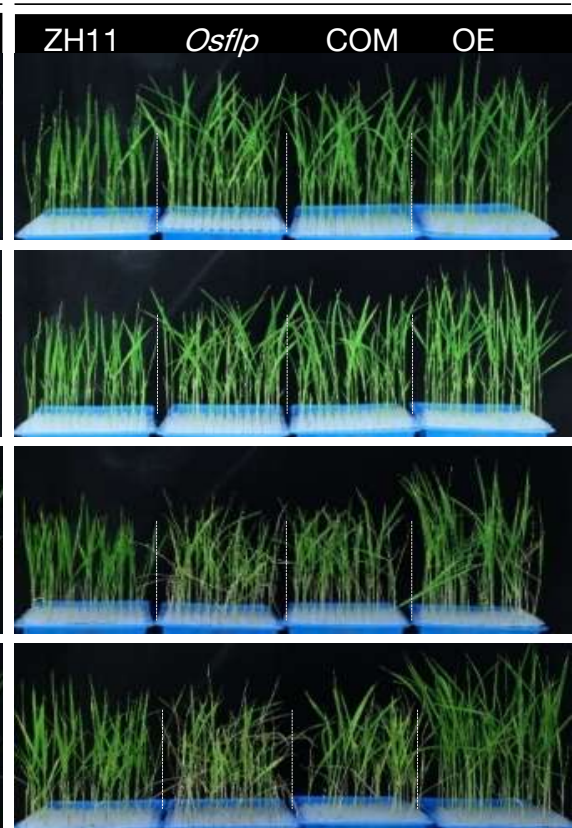

E

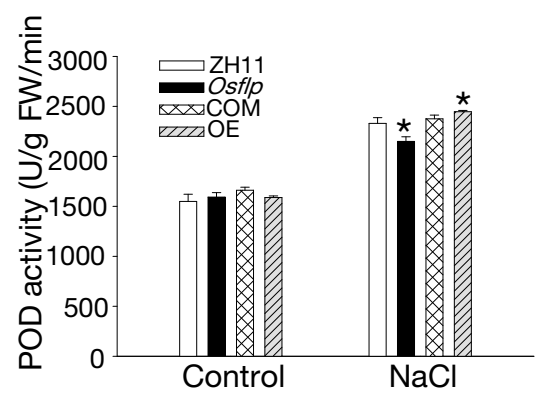

$\mathrm{H}$

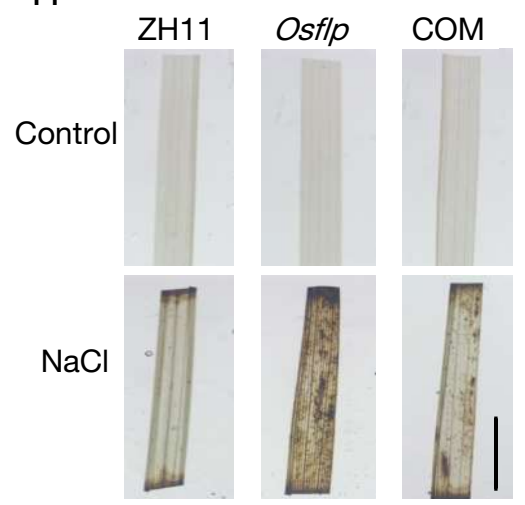

B

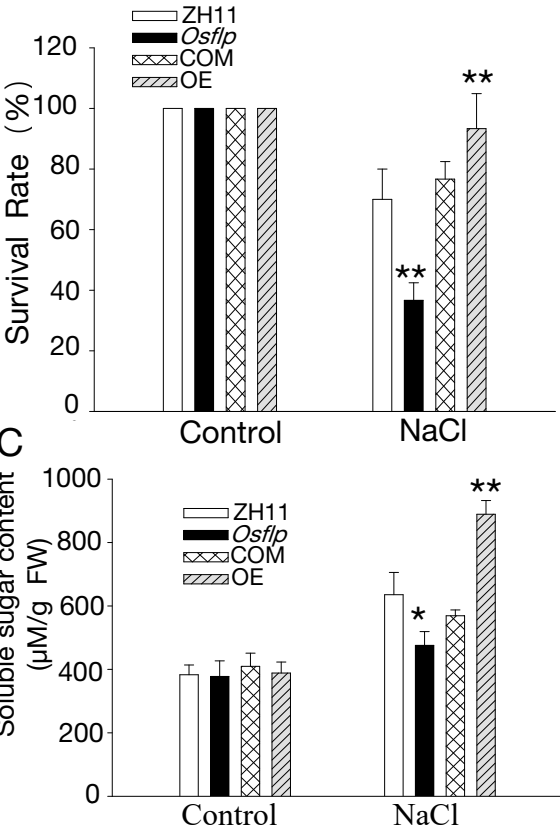

F

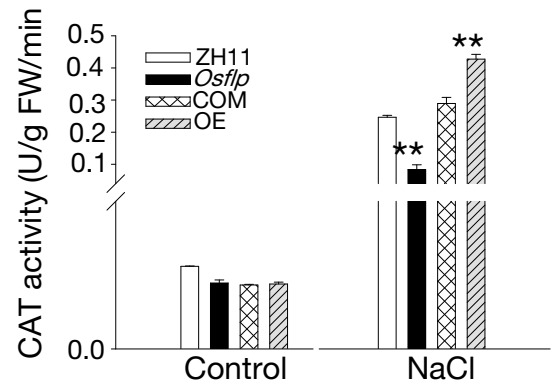

I

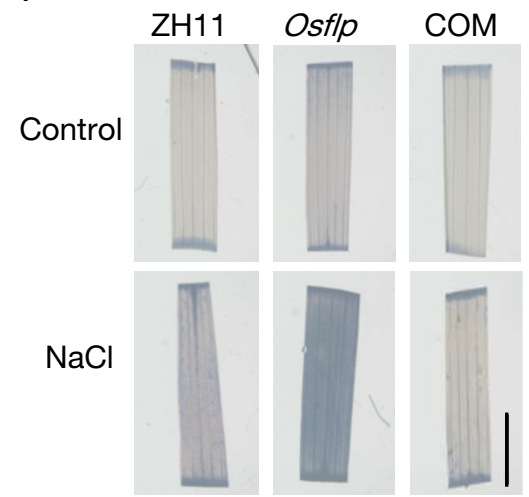


Fig. 5

A

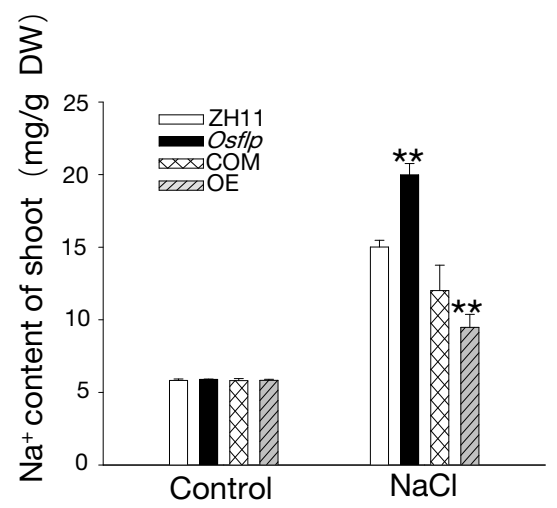

D

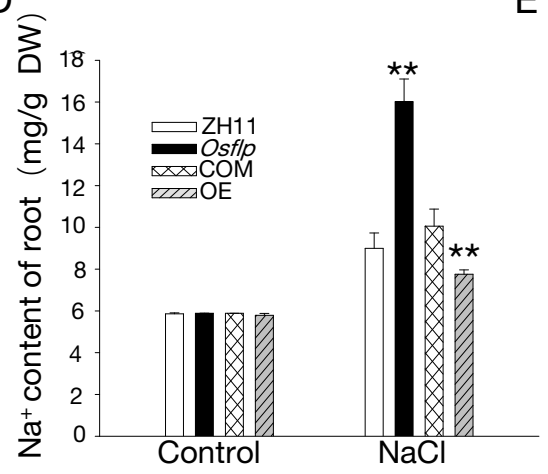

B
C
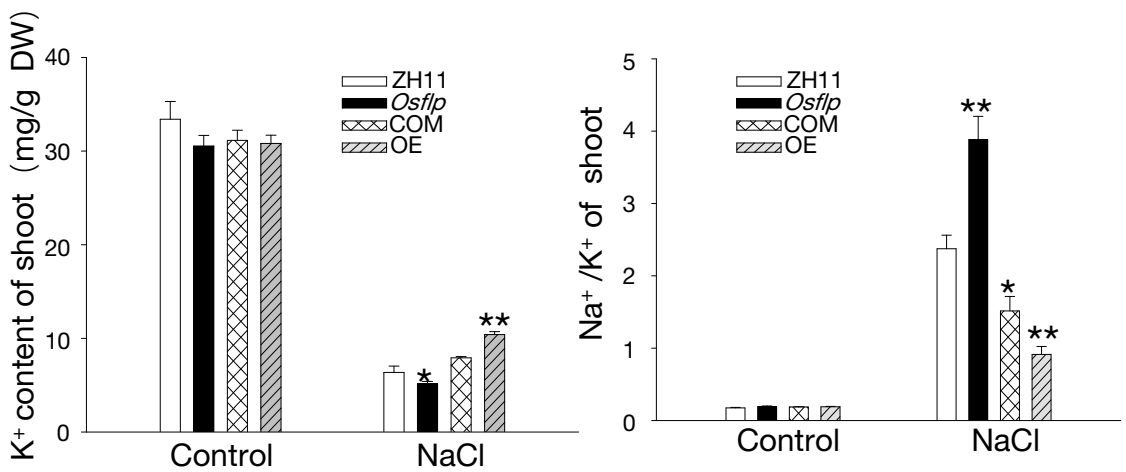

F

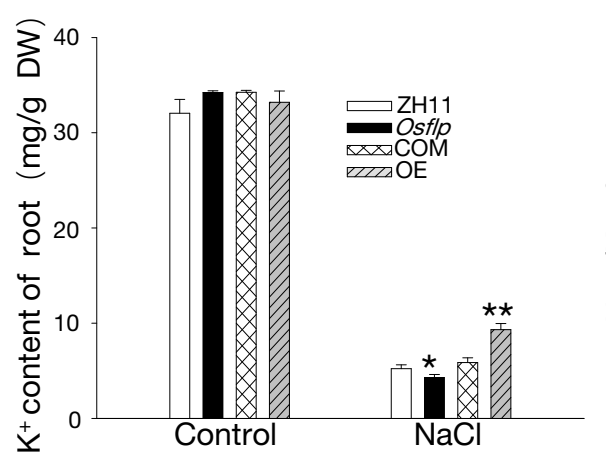

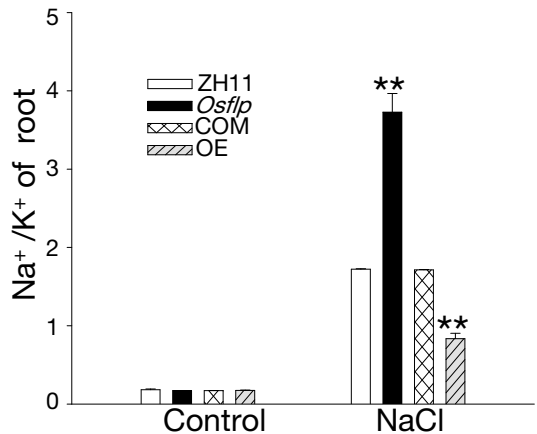


Fig. 6

A.

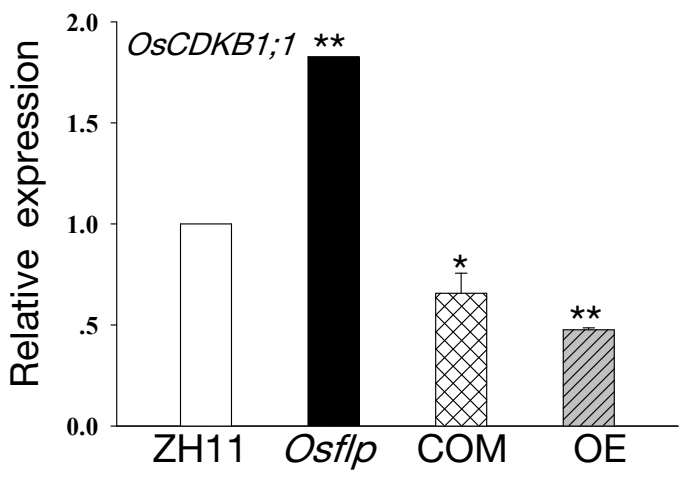

B.

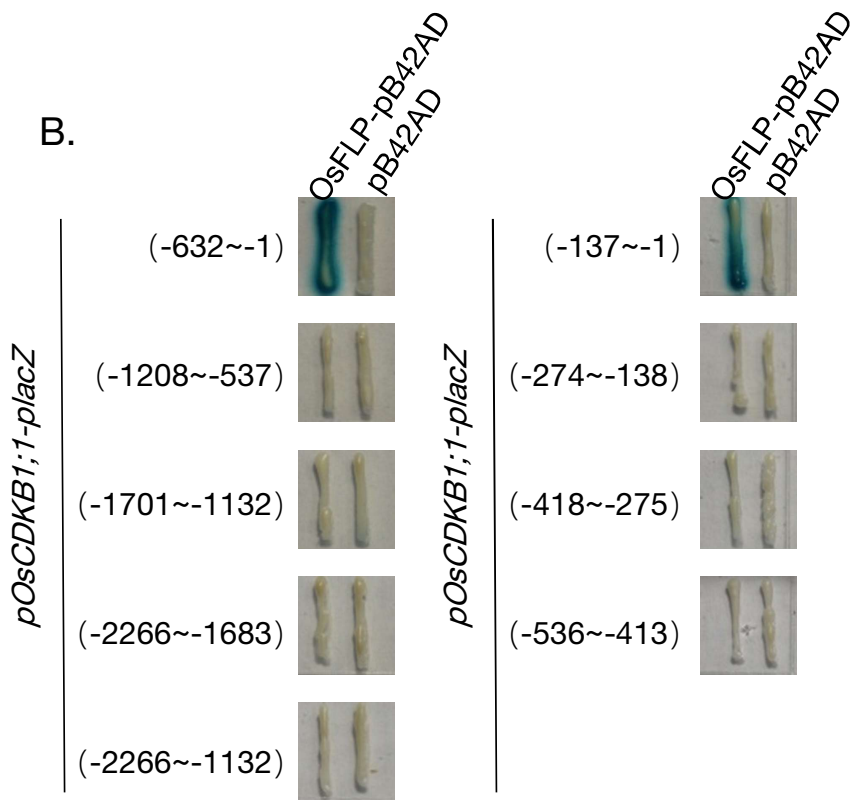

C.

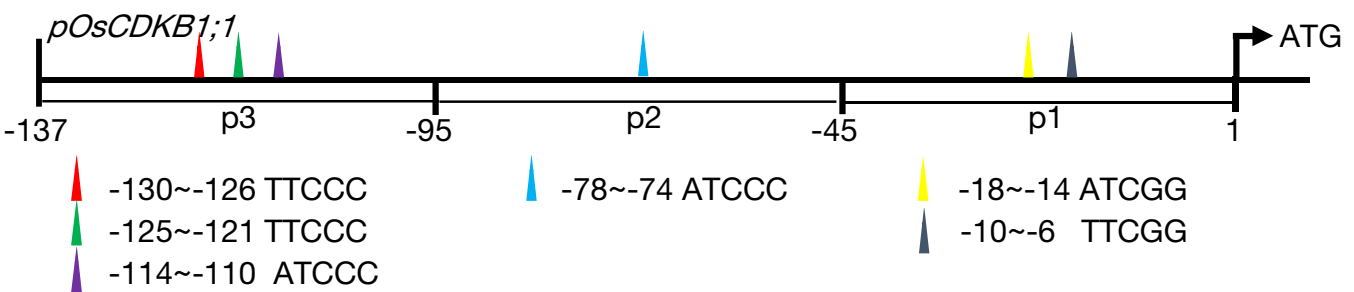

D.

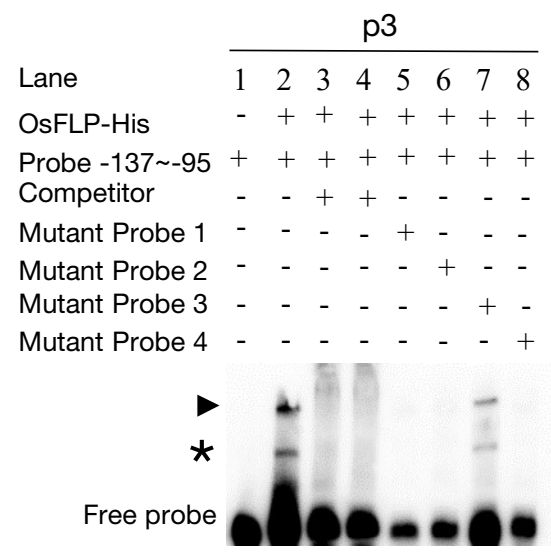

E.

Lane OsFLP-His

Probe -94 -45 Competitor Mutant Probe

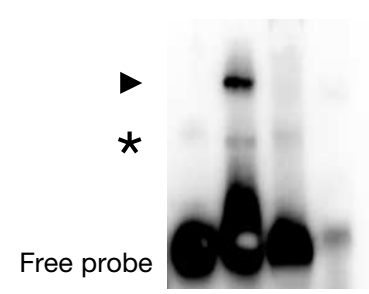

F.

Lane \begin{tabular}{llllll}
\multicolumn{1}{c}{$\mathrm{p} 1$} \\
\hline 1 & 2 & 3 & 4 & 5 & 6 \\
& + & + & + & + & +
\end{tabular} OsFLP-His - +++++ Probe -44 -1 ++++++ Competitor - -+ - Mutant Probe 1 - $\quad-\quad+\quad-$ Mutant Probe 2 - $\quad-\quad-\quad-\quad+$ Mutant Probe 3 - 
Fig. 7

A.

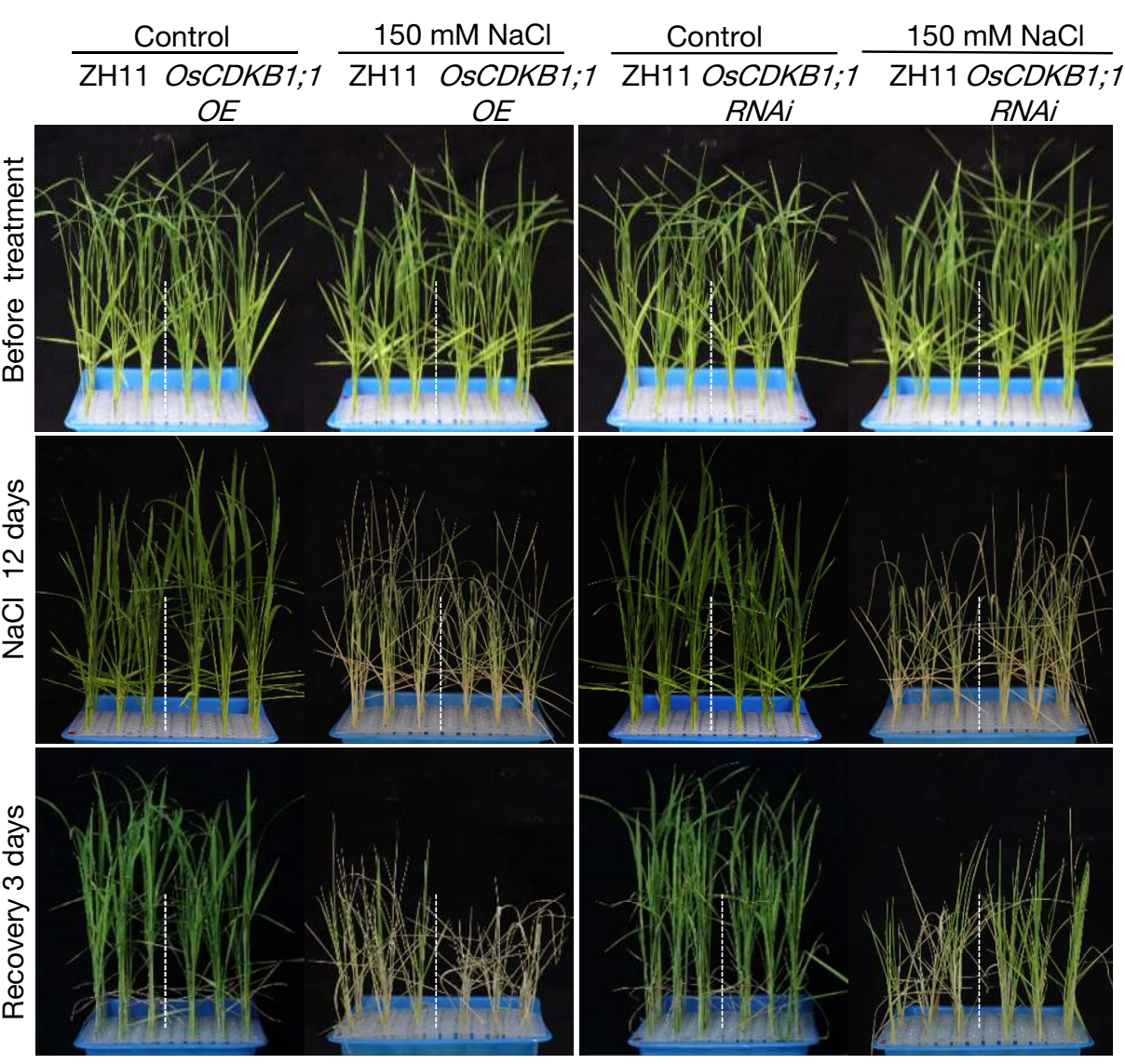

B.

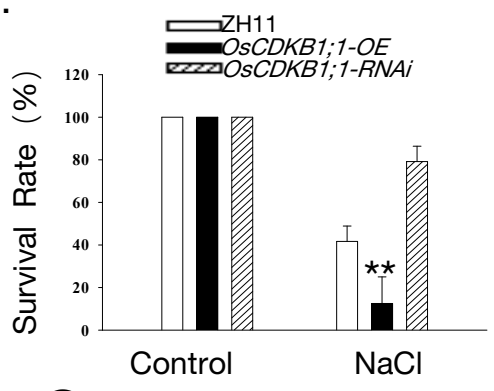

C. $\sum_{4}^{18} \square^{2 \mathrm{H} 11}$
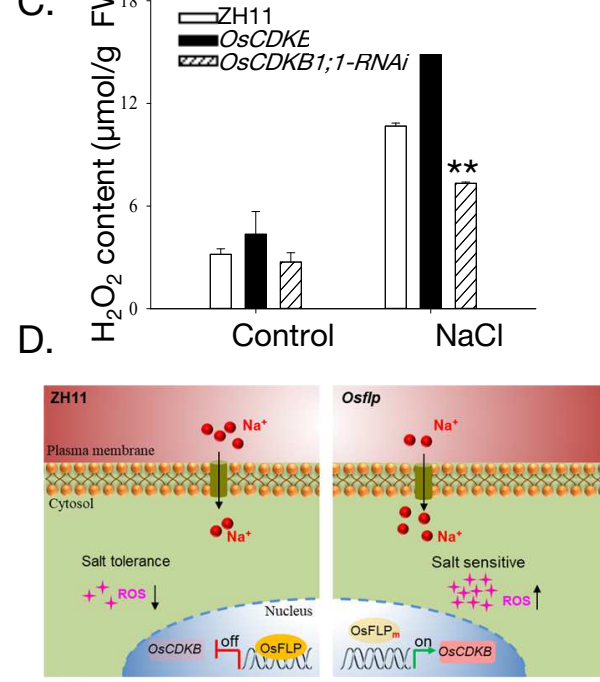


\section{Supplementary Files}

This is a list of supplementary files associated with this preprint. Click to download.

- DatasetS1.RNAseqOsflpvsZH11annotation.xlsx

- DatasetS2.RNAseqdataanalysiswithcategories.xls

- DatasetS3.GOanalysiswithRNAseqdata.xlsx

- DatasetS4.ChIPseqoriginaldataannotation.xls

- DatasetS5.KEGGEnrichmentwithChIPseqdata.xls

- DatasetS6.RNAseqandChIPseqintegrativeanalysis.xIsx

- DatasetS7.KEGGEnrichmentgenesfromRNAseqandChIPseq.xIsx

- Supplementaryfiguresandtables.pdf 